\title{
Pion-to-vacuum vector and axial vector amplitudes and weak decays of pions in a magnetic field
}

\author{
M. Coppola, ${ }^{1,2}$ D. Gomez Dumm, ${ }^{3}$ S. Noguera, ${ }^{4}$ and N. N. Scoccola ${ }^{1,2,5}$ \\ ${ }^{1}$ CONICET, Rivadavia 1917, 1033 Buenos Aires, Argentina \\ ${ }^{2}$ Physics Department, Comisión Nacional de Energía Atómica, Avenue Libertador 8250, \\ 1429 Buenos Aires, Argentina \\ ${ }^{3}$ IFLP, CONICET-Departamento de Física, Facultad de Ciencias Exactas, \\ Universidad Nacional de La Plata, C.C. 67, 1900 La Plata, Argentina \\ ${ }^{4}$ Departamento de Física Teórica and IFIC, Centro Mixto Universidad de Valencia-CSIC, \\ E-46100 Burjassot (Valencia), Spain \\ ${ }^{5}$ Universidad Favaloro, Solís 453, 1078 Buenos Aires, Argentina
}

(Received 26 October 2018; published 29 March 2019)

\begin{abstract}
We propose a model-independent parametrization for the one-pion-to-vacuum matrix elements of the vector and axial vector hadronic currents in the presence of an external uniform magnetic field. It is shown that, in general, these hadronic matrix elements can be written in terms of several gauge covariant Lorentz structures and form factors. Within this framework we obtain a general expression for the weak decay $\pi^{-} \rightarrow l \bar{\nu}_{l}$ and discuss the corresponding limits of strong and weak external magnetic fields.
\end{abstract}

DOI: 10.1103/PhysRevD.99.054031

\section{INTRODUCTION}

The effect of intense magnetic fields on the properties of strongly interacting matter has gained significant interest in recent years [1-3]. This is mostly motivated by the realization that strong magnetic fields might play an important role in the study of the early Universe [4], in the analysis of high energy noncentral heavy ion collisions [5], and in the description of physical systems such as compact stellar objects called magnetars [6]. It is well known that magnetic fields also induce interesting phenomena like the enhancement of the QCD vacuum (the so-called "magnetic catalysis") [7], and the decrease of critical temperatures for chiral restoration and deconfinement QCD transitions [8].

In this work we concentrate on the effect of a magnetic field $\vec{B}$ on the weak pion-to-lepton decay $\pi^{-} \rightarrow l \bar{\nu}_{l}$. In fact, the study of weak decays of hadrons in the presence of strong electromagnetic fields has a rather long history. Already in the mid 1960s, the effect of some particularly simple electromagnetic field configurations on leptonic decays of charged pions was considered $[9,10]$. Some years later, the decay of a neutron in the presence of a magnetic field was also studied [11,12]. In most of the

Published by the American Physical Society under the terms of the Creative Commons Attribution 4.0 International license. Further distribution of this work must maintain attribution to the author(s) and the published article's title, journal citation, and DOI. Funded by SCOAP . existing calculations of the decay rates, however, the effect of the external field on the internal structure of the participating particles has not been taken into account. Only in recent years has the $B$-dependence of pion masses actively been investigated from the theoretical point of view. This has been done using approaches like e.g., chiral perturbation theory $[13,14]$, quark effective models [15-26] and lattice QCD (LQCD) simulations [8,27,28]. In addition, some of these works [13-21] (see also Refs. [29,30]) considered, for the case of the neutral pion, the $B$-dependence of the decay constant $f_{\pi^{0}}$. For the charged pion, such an effect has been analyzed in the context of chiral perturbation theory [14], quark-antiquark effective chiral models [30] and very recently through LQCD calculations [31]. An interesting observation was made in Ref. [29], where it is claimed that, due to the explicit breaking of rotational invariance caused by the magnetic field, one can define two different decay constants, one of them associated with the direction parallel to $\vec{B}$ and another one associated with the perpendicular directions. A further relevant statement has been pointed out in Ref. [31]. In that work it is noted that the existence of the background field opens the possibility of a nonzero pion-to-vacuum transition via the vector piece of the electroweak current, which implies the existence of a further decay constant. Taking into account this new constant, the authors of Ref. [31] obtained an expression for the decay width under the assumption that the decaying pion is at rest. However, one should note that in the case of a charged pion this condition cannot be exactly fulfilled, 
due to the presence of the magnetic field. Even in its lowest energy level (hence, in the lowest Landau level), the charged pion still keeps a zero point motion. In this context, the aim of the present work is twofold. Firstly, considering an external uniform magnetic field, we determine the form of the most general model-independent hadronic matrix elements, written in terms of gauge covariant Lorentz structures. In particular, we show that in the presence of a magnetic field several independent form factors can be in principle defined. Secondly, given the general form of the hadronic matrix elements, we obtain an expression for the $\pi^{-} \rightarrow l \bar{\nu}_{l}$ decay width, taking into account the effect of the magnetic field on both pion and lepton wave functions.

This paper is organized as follows. In Sec. II we discuss the structure of the pion-to-vacuum matrix elements in the presence of a uniform static magnetic field from a general point of view. We start by identifying the relevant gauge covariant Lorentz structures, and then we proceed to obtain the hadronic matrix elements for neutral and charged pions. In Sec. III we obtain an explicit expression for the $\pi^{ \pm}$ leptonic weak decay width. After considering the general case of an arbitrary magnetic field strength, we discuss the particular cases of both strong and weak magnetic fields. Finally, in Sec. IV we present our main conclusions. We also include Appendixes A, B, C and D to quote technical details of our calculations.

\section{PION-TO-VACUUM AMPLITUDES IN THE PRESENCE OF AN EXTERNAL UNIFORM MAGNETIC FIELD: GENERAL STRUCTURE}

In this section we analyze the general form of the pionto-vacuum matrix elements of vector and axial vector quark currents. Throughout this work we use the Minkowski metric $g^{\mu \nu}=\operatorname{diag}(1,-1,-1,-1)$, as well as the convention $\epsilon^{0123}=+1$ for the totally antisymmetric tensor $\epsilon^{\mu \nu \alpha \beta}$.

Let us start by considering the hadronic matrix elements for the case of a neutral pion in the absence of the external magnetic field. The amplitudes for the vector and axial vector quark currents are

$$
\begin{aligned}
& H_{V}^{0, \mu}(x, \vec{p})=\left\langle 0\left|\bar{\psi}(x) \gamma^{\mu} \frac{\tau_{3}}{2} \psi(x)\right| \pi^{0}(\vec{p})\right\rangle, \\
& H_{A}^{0, \mu}(x, \vec{p})=\left\langle 0\left|\bar{\psi}(x) \gamma^{\mu} \gamma_{5} \frac{\tau_{3}}{2} \psi(x)\right| \pi^{0}(\vec{p})\right\rangle,
\end{aligned}
$$

where $\psi(x)$ includes the $u$ and $d$ quark fields,

$$
\psi(x)=\left(\begin{array}{l}
\psi_{u}(x) \\
\psi_{d}(x)
\end{array}\right)
$$

and $\tau_{3}$ is a Pauli matrix that acts on flavor space. To deal with the matrix elements in Eq. (1) it is possible to hadronize the quark currents, i.e., to consider matrix elements of hadronic field operators carrying appropriate
Lorentz indices and quantum numbers. In the low energy limit (typically, below the $\rho$ meson threshold), the relevant hadronic field is the pion field $\phi_{\pi^{0}}(x)$, and (in absence of external fields) the only available vectorlike differential operator is the momentum operator $\hat{p}^{\mu}=i \partial^{\mu}$. Since the pion field is pseudoscalar, only the matrix element of the axial-vector hadronic current can be nonzero. In this way, one has

$$
\begin{aligned}
\left\langle 0\left|\bar{\psi}(x) \gamma^{\mu} \frac{\tau_{3}}{2} \psi(x)\right| \pi^{0}(\vec{p})\right\rangle & =0, \\
\left\langle 0\left|\bar{\psi}(x) \gamma^{\mu} \gamma_{5} \frac{\tau_{3}}{2} \psi(x)\right| \pi^{0}(\vec{p})\right\rangle & =f\left(\hat{p}^{2}\right) \partial^{\mu}\left\langle 0\left|\phi_{\pi^{0}}(x)\right| \pi^{0}(\vec{p})\right\rangle .
\end{aligned}
$$

Here, the function $f\left(\hat{p}^{2}\right)$ contains all the information of nonperturbative QCD contributions. Using the explicit form of $\phi_{\pi^{0}}(x)$ and the commutation rules for the corresponding creation and annihilation operators [see Eqs. (A1) and (A2)], one immediately finds

$$
\begin{aligned}
& H_{V}^{0, \mu}(x, \vec{p})=0 \\
& H_{A}^{0, \mu}(x, \vec{p})=-i f\left(p^{2}\right) p^{\mu} e^{-i p \cdot x} .
\end{aligned}
$$

As usual, the four-momentum $p^{\mu}$ is defined by $p^{\mu}=$ $\left(E_{\pi}, \vec{p}\right)$, with $E_{\pi}=\sqrt{m_{\pi}^{2}+|\vec{p}|^{2}}$. Similar expressions can be obtained for charged pions. It can be seen that the invariance of strong interactions under $\mathcal{P}, \mathcal{C}$ and $\mathcal{T}$ transformations implies that $f\left(p^{2}\right)$ is a real function. In the absence of external fields, the pion decay constant is given by $f_{\pi}=f\left(m_{\pi}^{2}\right) \simeq 92.3 \mathrm{MeV}$ [32].

We turn now to the situation in which a static external electromagnetic field is present. In this case, other tensor structures are possible. For a particle of charge $Q$ the relevant basic tensors are the gauge covariant derivative $\mathcal{D}^{\mu}$ and the gauge invariant electromagnetic field strength $F^{\mu \nu}$, defined as

$$
\mathcal{D}^{\mu}=\partial^{\mu}+i Q \mathcal{A}^{\mu}, \quad F^{\mu \nu}=\partial^{\mu} \mathcal{A}^{\nu}-\partial^{\nu} \mathcal{A}^{\mu} .
$$

Taking them as building blocks, one can in principle obtain an infinite number of differential operators with different Lorentz tensor structures. However, for the particular case of a uniform static magnetic field $\vec{B}$, it is well known (see e.g., Ref. [33]) that only a few independent tensors exist. Noting that $F^{0 i}=0$ and $F^{i j}=F_{i j}=-\epsilon_{i j k} B^{k}$, we get

$$
\left[\mathcal{D}^{\mu}, \mathcal{D}^{\nu}\right]=i Q F^{\mu \nu}=-i Q \epsilon^{0 \mu \nu k} B^{k}, \quad k=1,2,3 .
$$

For definiteness, and without losing generality, in what follows we take $B^{k}=B \delta_{k 3}$. Using the above relations it is easy to prove that one has only two independent scalars, apart from the particle electric charge $Q$ and $F^{\mu \nu} F_{\mu \nu}=2 B^{2}$. These can be taken to be 
$\mathcal{D}_{\|}^{2}=\left(\mathcal{D}^{3}\right)^{2}-\left(\mathcal{D}^{0}\right)^{2}, \quad \mathcal{D}_{\perp}^{2}=-\left(\mathcal{D}^{1}\right)^{2}-\left(\mathcal{D}^{2}\right)^{2}$.

In addition, it is possible to find four independent fourvectors. One possible choice is the set

$$
\begin{aligned}
\mathcal{D}^{\mu} & =\left(\mathcal{D}^{0}, \overrightarrow{\mathcal{D}}\right), \\
-i F^{\mu \nu} \mathcal{D}_{\nu} & =-i B\left(0, \mathcal{D}^{2},-\mathcal{D}^{1}, 0\right), \\
F^{\mu \nu} F_{\nu \alpha} \mathcal{D}^{\alpha} & =-B^{2}\left(0, \mathcal{D}^{1}, \mathcal{D}^{2}, 0\right), \\
\frac{1}{2} \epsilon^{\mu \nu \alpha \beta} F_{\nu \alpha} \mathcal{D}_{\beta} & =B\left(\mathcal{D}^{3}, 0,0, \mathcal{D}^{0}\right)
\end{aligned}
$$

(notice that the last of these tensors transforms in fact as an axial vector).

From the above expressions for Lorentz scalars and fourvectors, we can write now a general form for the hadronic currents we are interested in. We consider first the case of the neutral pion, for which $Q=0$ and the operator $\mathcal{D}^{\mu}$ reduces to the usual derivative $\partial^{\mu}$. Taking into account once again the intrinsic parity of the pion field, one has

$$
\begin{aligned}
&\left\langle 0\left|\bar{\psi}(x) \gamma^{\mu} \frac{\tau_{3}}{2} \psi(x)\right| \pi^{0}(\vec{p})\right\rangle \hat{f}_{\pi^{0}}^{(V)}\left(\partial^{3}, 0,0, \partial^{0}\right)\left\langle 0\left|\phi_{\pi^{0}}(x)\right| \pi^{0}(\vec{p})\right\rangle, \\
&\left\langle 0\left|\bar{\psi}(x) \gamma^{\mu} \gamma_{5} \frac{\tau_{3}}{2} \psi(x)\right| \pi^{0}(\vec{p})\right\rangle \\
&=\left[\hat{f}_{\pi^{0}}^{(A 1)}\left(\partial^{0}, \vec{\partial}\right)-i \hat{f}_{\pi^{0}}^{(A 2)}\left(0, \partial^{2},-\partial^{1}, 0\right)\right. \\
&\left.\quad-\hat{f}_{\pi^{0}}^{(A 3)}\left(0, \partial^{1}, \partial^{2}, 0\right)\right]\left\langle 0\left|\phi_{\pi^{0}}(x)\right| \pi^{0}(\vec{p})\right\rangle,
\end{aligned}
$$

where $\hat{f}_{\pi^{0}}^{(V)}$ and $\hat{f}_{\pi^{0}}^{(A i)}$ are complex functions of the magnetic field and the scalar differential operators $\partial_{\|}^{2}$ and $\partial_{\perp}^{2}$. The hadronic matrix elements can be readily obtained using Eqs. (A1) and (A2). We find it convenient to define some linear combinations of the respective Lorentz components, namely

$$
\begin{gathered}
H_{\|, V}^{0, \epsilon}(x, \vec{p}) \equiv H_{V}^{0,0}(x, \vec{p})+\epsilon H_{V}^{0,3}(x, \vec{p}) \\
=-i \epsilon f_{\pi^{0}}^{(V)}\left(E_{\pi^{0}}+\epsilon p^{3}\right) e^{-i p \cdot x}, \\
H_{\perp, V}^{0, \epsilon}(x, \vec{p}) \equiv H_{V}^{0,1}(x, \vec{p})+i \epsilon H_{V}^{0,2}(x, \vec{p})=0,
\end{gathered}
$$

and

$$
\begin{gathered}
H_{\|, A}^{0, \epsilon}(x, \vec{p}) \equiv H_{A}^{0,0}(x, \vec{p})+\epsilon H_{A}^{0,3}(x, \vec{p}) \\
=-i f_{\pi^{0}}^{(A 1)}\left(E_{\pi^{0}}+\epsilon p^{3}\right) e^{-i p \cdot x}, \\
H_{\perp, A}^{0, \epsilon}(x, \vec{p}) \equiv H_{A}^{0,1}(x, \vec{p})+i \epsilon H_{A}^{0,2}(x, \vec{p}) \\
=-i\left[f_{\pi^{0}}^{(A 1)}-\epsilon f_{\pi^{0}}^{(A 2)}-f_{\pi^{0}}^{(A 3)}\right]\left(p^{1}+i \epsilon p^{2}\right) e^{-i p \cdot x},
\end{gathered}
$$

with $\epsilon= \pm$. Here, $f_{\pi^{0}}^{(V)}$ and $f_{\pi^{0}}^{(A i)}$ are functions of $p_{\perp}^{2}=$ $\left(p^{1}\right)^{2}+\left(p^{2}\right)^{2}$ and $p_{\|}^{2}=E_{\pi^{0}}^{2}-\left(p^{3}\right)^{2}$, with $p_{\|}^{2}-p_{\perp}^{2}=$ $p^{2}=m_{\pi^{0}}^{2}$. Notice that, although it is not indicated explicitly, the pion mass $m_{\pi^{0}}$ is a function of the magnetic field $B$. As in the $B=0$ case, it is important to consider the constraints on the form factors arising from the discrete symmetries of the interaction Lagrangian in the presence of the magnetic field. This is discussed in some detail in Appendix B, where it is shown that these symmetries lead to $f_{\pi^{0}}^{(A 2)}=0$ while the remaining form factors turn out to be real. In this way, we conclude that the most general forms of the vector and axial vector pion-to-vacuum matrix elements, in the presence of an external constant and homogenous magnetic field along the 3 -axis, are

$$
\begin{gathered}
H_{\|, V}^{0, \epsilon}(x, \vec{p})=-i \epsilon f_{\pi^{0}}^{(V)}\left(E_{\pi^{0}}+\epsilon p^{3}\right) e^{-i p \cdot x}, \\
H_{\perp, V}^{0, \epsilon}(x, \vec{p})=0
\end{gathered}
$$

and

$$
\begin{gathered}
H_{\|, A}^{0, \epsilon}(x, \vec{p})=-i f_{\pi^{0}}^{(A 1)}\left(E_{\pi^{0}}+\epsilon p^{3}\right) e^{-i p \cdot x}, \\
H_{\perp, A}^{0, \epsilon}(x, \vec{p})=-i\left[f_{\pi^{0}}^{(A 1)}-f_{\pi^{0}}^{(A 3)}\right]\left(p^{1}+i \epsilon p^{2}\right) e^{-i p \cdot x},
\end{gathered}
$$

where all form factors are real. The results in Eqs. (17) and (18) are in agreement with the observation made in Ref. [29] that, due to the explicit breaking of rotational invariance caused by the magnetic field, one can define for the neutral meson two different form factors related to the axial current. One of them can be associated with the direction parallel to $\vec{B}$, and the other one with the perpendicular directions. In addition, according to Eq. (15) we find that a further form factor related to the vector current can be defined as well.

Let us consider now the case of the charged pion $\pi^{\sigma}$, with $\sigma= \pm$ (electric charge $Q=\sigma|e|)$. We adopt in this work the Landau gauge, in which $\mathcal{A}^{\mu}=\left(0,0, B x^{1}, 0\right)$. Then, $\mathcal{D}^{\mu}=\partial^{\mu}+i s B_{e} x^{1} \delta_{\mu 2}$, with $s=\operatorname{sign}(\sigma B)$ and $B_{e}=|e B|$. One has in this case

$$
\begin{aligned}
H_{V}^{\sigma, \mu}(x, \breve{p}) & =\left\langle 0\left|\bar{\psi}(x) \gamma^{\mu} \tau^{-\sigma} \psi(x)\right| \pi^{\sigma}(\breve{p})\right\rangle \\
& =\sqrt{2}\left(\mathcal{D}^{3}, 0,0, \mathcal{D}^{0}\right) \hat{f}_{\pi^{\sigma}}^{(V)}\left\langle 0\left|\phi_{\pi^{\sigma}}^{s}(x)\right| \pi^{\sigma}(\breve{p})\right\rangle
\end{aligned}
$$

and

$$
\begin{aligned}
H_{A}^{\sigma, \mu}(x, \breve{p})= & \left\langle 0\left|\bar{\psi}(x) \gamma^{\mu} \gamma_{5} \tau^{-\sigma} \psi(x)\right| \pi^{\sigma}(\breve{p})\right\rangle \\
= & \sqrt{2}\left[\left(\mathcal{D}^{0}, \overrightarrow{\mathcal{D}}\right) \hat{f}_{\pi^{\sigma}}^{(A 1)}-i s\left(0, \mathcal{D}^{2},-\mathcal{D}^{1}, 0\right) \hat{f}_{\pi^{\sigma}}^{(A 2)}\right. \\
& \left.-\left(0, \mathcal{D}^{1}, \mathcal{D}^{2}, 0\right) \hat{f}_{\pi^{\sigma}}^{(A 3)}\right]\left\langle 0\left|\phi_{\pi^{\sigma}}^{s}(x)\right| \pi^{\sigma}(\breve{p})\right\rangle,
\end{aligned}
$$


where $\tau^{ \pm}=\left(\tau_{1} \pm i \tau_{2}\right) / 2$. Here, $\hat{f}_{\pi^{\sigma}}^{(V)}$ and $\hat{f}_{\pi^{\sigma}}^{(A i)}$ are functions of the scalar operators $\mathcal{D}_{\|}^{2}$ and $\mathcal{D}_{\perp}^{2}$, while $\breve{p}=\left(\ell, p^{2}, p^{3}\right), \ell$ being a non-negative integer (Landau index), denotes a set of quantum numbers that characterize the charged pion state in the presence of the magnetic field (see Appendix A.2 for details). The sign $s$ in the term carrying $\hat{f}_{\pi^{\sigma}}^{(A 2)}$ has been conventionally introduced for later convenience. Notice that in Eqs. (19) and (20) the operators $\hat{f}_{\pi^{\sigma}}^{(V)}$ and $\hat{f}_{\pi^{\sigma}}^{(A i)}$ have been placed to the right of the operators listed in Eq. (8). In fact, the ordering is relevant, since (contrary to the case of the neutral pion) the operators $\mathcal{D}^{1}$ and $\mathcal{D}^{2}$ do not commute. On the other hand, since the basis formed by the operators in Eq. (8) is complete, our ordering choice does not imply a loss of generality (different orderings just correspond to alternative definitions of the form factors). The convenience of our election will become clear below.

To proceed we need the general form of the $\pi^{\sigma}$ wave functions in the presence of the external magnetic field. For our choice of gauge and magnetic field direction these are given by Eq. (A10). From this equation, together with the commutation relations of the associated creation and annihilation operators given in Eq. (A16), one can obtain general expressions for the hadronic matrix elements. As in the case of the neutral pion, these can be conveniently written in terms of linear combinations of their Lorentz components. We have

$$
\begin{aligned}
& H_{\|, V}^{\sigma, \epsilon}(x, \breve{p}) \equiv H_{V}^{\sigma, 0}(x, \breve{p})+\epsilon H_{V}^{\sigma, 3}(x, \breve{p})=\epsilon \sqrt{2} \mathcal{D}_{\|}^{\epsilon} \hat{f}_{\pi^{\sigma}}^{(V)} \mathbb{F}_{\bar{p}}^{s}(x), \\
& H_{\perp, V}^{\sigma, \epsilon}(x, \breve{p}) \equiv H_{V}^{\sigma, 1}(x, \breve{p})+i \epsilon H_{V}^{\sigma, 2}(x, \breve{p})=0
\end{aligned}
$$

and

$$
\begin{aligned}
H_{\|, A}^{\sigma, \epsilon}(x, \breve{p}) & \equiv H_{A}^{\sigma, 0}(x, \breve{p})+\epsilon H_{A}^{\sigma, 3}(x, \breve{p})=\sqrt{2} \mathcal{D}_{\|}^{\epsilon} \hat{f}_{\pi^{\sigma}}^{(A 1)} \mathbb{F}_{\bar{p}}^{S}(x), \\
H_{\perp, A}^{\sigma, \epsilon}(x, \breve{p}) & \equiv H_{A}^{\sigma, 1}(x, \breve{p})+i \epsilon H_{A}^{\sigma, 2}(x, \breve{p}) \\
& =\sqrt{2} \mathcal{D}_{\perp}^{\epsilon}\left(\hat{f}_{\pi^{\sigma}}^{(A 1)}-s \epsilon \hat{f}_{\pi^{\sigma}}^{(A 2)}-\hat{f}_{\pi^{\sigma}}^{(A 3)}\right) \mathbb{F}_{\bar{p}}^{S}(x),
\end{aligned}
$$

where $\quad \mathcal{D}_{\|}^{\epsilon}=\mathcal{D}^{0}+\epsilon \mathcal{D}^{3} \quad$ and $\quad \mathcal{D}_{\perp}^{\epsilon}=\mathcal{D}^{1}+i \epsilon \mathcal{D}^{2}$. We use here the notation $\bar{p}=\left(E_{\pi^{\sigma}}, \breve{p}\right)$, with $E_{\pi^{\sigma}}=$ $\sqrt{m_{\pi^{\sigma}}^{2}+(2 \ell+1) B_{e}+\left(p^{3}\right)^{2}}$ (in fact, one has $E_{\pi^{+}}=E_{\pi^{-}}$, the charge index $\sigma$ being kept in order to distinguish $E_{\pi^{ \pm}}$from $E_{\pi^{0}}$ ). The functions $\mathbb{F}_{\bar{p}}^{ \pm}(x)$ are eigenfunctions of the charged pion Klein-Gordon equation in the presence of the magnetic field, for our choices of gauge and magnetic field direction. Their explicit expressions are given in Eq. (A12). Notice that on the rhs of Eqs. (21) and (22) we have differential operators acting on these functions. Using the relations

$$
\mathcal{D}_{\|}^{\epsilon} \mathbb{F}_{\bar{p}}^{s}(x)=-i\left(E_{\pi^{\sigma}}+\epsilon p^{3}\right) \mathbb{F}_{\bar{p}}^{s}(x),
$$

$$
\mathcal{D}_{\perp}^{\epsilon} \mathbb{F}_{\bar{p}}^{s}(x)=-s \epsilon \sqrt{B_{e}(2 \ell+1-s \epsilon)} \mathbb{F}_{\bar{p}-s \epsilon}^{s}(x)
$$

and

$$
\begin{aligned}
& \mathcal{D}_{\|\|}^{2} \mathbb{F}_{\bar{p}}^{s}(x)=\left[E_{\pi^{\sigma}}^{2}-\left(p^{3}\right)^{2}\right] \mathbb{F}_{\bar{p}}^{s}(x), \\
& \mathcal{D}_{\perp}^{2} \mathbb{F}_{\bar{p}}^{s}(x)=(2 \ell+1) B_{e} \mathbb{F}_{\bar{p}}^{s}(x),
\end{aligned}
$$

where $\bar{p} \pm 1 \equiv\left(E_{\pi^{\sigma}}, \breve{p} \pm 1\right)$, with $\breve{p} \pm 1=\left(\ell \pm 1, p^{2}, p^{3}\right)$, we finally obtain

$$
\begin{gathered}
H_{\|, V}^{\sigma, \epsilon}(x, \breve{p})=-i \epsilon \sqrt{2} f_{\pi^{\sigma}}^{(V)}\left(E_{\pi^{\sigma}}+\epsilon p^{3}\right) \mathbb{F}_{\bar{p}}^{s}(x), \\
H_{\perp, V}^{\sigma, \epsilon}(x, \breve{p})=0
\end{gathered}
$$

and

$$
\begin{gathered}
H_{\|, A}^{\sigma, \epsilon}(x, \breve{p})=-i \sqrt{2} f_{\pi^{\sigma}}^{(A 1)}\left(E_{\pi^{\sigma}}+\epsilon p^{3}\right) \mathbb{F}_{\bar{p}}^{s}(x), \\
H_{\perp, A}^{\sigma, \epsilon}(x, \breve{p})=-s \epsilon \sqrt{2}\left(f_{\pi^{\sigma}}^{(A 1)}-s \epsilon f_{\pi^{\sigma}}^{(A 2)}-f_{\pi^{\sigma}}^{(A 3)}\right) \\
\times \sqrt{B_{e}(2 \ell+1-s \epsilon)} \mathbb{F}_{\bar{p}-s \epsilon}^{s}(x) .
\end{gathered}
$$

In the above expressions, the form factors $f_{\pi^{\sigma}}^{(V)}, f_{\pi^{\sigma}}^{(A i)}$ arise from the action of the operators $\hat{f}_{\pi^{\sigma}}^{(V)}, \hat{f}_{\pi^{\sigma}}^{(A i)}$ on the functions $\mathbb{F}_{\bar{p}}^{s}(x)$. These operators are, in general, gauge-dependent functions of the operators $\mathcal{D}_{\|}^{2}$ and $\mathcal{D}_{\perp}^{2}$. However, for an onenergy-shell pion, taking into account Eqs. (25) and (26) it can be seen that the resulting form factors $f_{\pi^{\sigma}}^{(V)}$ and $f_{\pi^{\sigma}}^{(A i)}$ turn out to be gauge-independent functions of the pion mass, $m_{\pi^{\sigma}}$; the third component of the momentum, $p^{3}$; the Landau index, $\ell$; and the magnetic field, $B$ (both explicitly and through the charged pion mass). In the so-called symmetric gauge, Eqs. (25) and (26) should be modified by changing the functions $\mathbb{F}_{\bar{p}}^{\sigma}(x)$ by the corresponding ones in that gauge, which involve associated Laguerre polynomials [34]. On the other hand, notice that the eigenvalues in Eqs. (25) and (26) are functions of $|B|$, while the scalar combination $F^{\mu \nu} F_{\mu \nu}$ depends on $B^{2}$. Therefore, taking into account the form of the four-vectors in Eq. (8), and the factor $s$ introduced in the term carrying $\hat{f}_{\pi^{\sigma}}^{(A 2)}$ in Eq. (20), it is seen that the vector form factor $f_{\pi^{\sigma}}^{(V)}$ should be odd under the exchange $B \rightarrow-B$, while the axial vector form factors $f_{\pi^{\sigma}}^{(A i)}$ should be even functions of $B$. For definiteness, in what follows we will take $B>0$, and consequently $s=\sigma$.

As in the case of the neutral pion, the discrete symmetries of the interaction Lagrangian in the presence of the magnetic field lead to some restrictions on the form factors $f_{\pi^{\sigma}}^{(V)}$ and $f_{\pi^{\sigma}}^{(A i)}$. Indeed, as shown in Appendix B, they have to be real and independent of the sign of the pion charge. Finally, let us point out that the form factors $f_{\pi^{\sigma}}^{(A 1)}$ appearing 
in Eqs. (29) and (30) are the same. In fact, this is a consequence of our choice of ordering in Eq. (20). Had we put the operator functions $\hat{f}_{\pi^{\sigma}}^{(A i)}$ to the left of the other operators in Eq. (20), the form factor $f_{\pi^{\sigma}}^{(A 1)}$ in Eq. (29) would have arisen from the action of $\hat{f}_{\pi^{\sigma}}^{(A 1)}$ on $\mathbb{F}_{\bar{p}}^{\sigma}(x)$, while the one in Eq. (30) would correspond to the action of $\hat{f}_{\pi^{\sigma}}^{(A 1)}$ on $\mathbb{F}_{\bar{p}-\sigma \epsilon}^{\sigma}(x)$. In any case, it should be stressed that this would also imply a redefinition of $f_{\pi^{\sigma}}^{(A 2)}$ and $f_{\pi^{\sigma}}^{(A 3)}$, in such a way that the contribution of $H_{\perp, A}^{\sigma, \epsilon}(x, \breve{p})$ to any physical quantity remains unchanged.

\section{WEAK DECAY WIDTH OF CHARGED PIONS UNDER A MAGNETIC FIELD}

\section{A. Basic relations and decay kinematics}

Let us analyze the decay width for the process $\pi^{-} \rightarrow l \bar{\nu}_{l}$, with $l=\mu, e$, in the presence of the external magnetic field. Following the notation introduced in the previous section, the initial charged pion state is determined by the quantum numbers $\breve{p}=\left(\ell, p^{2}, p^{3}\right)$, the associated energy being $E_{\pi^{-}}=\sqrt{m_{\pi^{-}}^{2}+(2 \ell+1) B_{e}+\left(p^{3}\right)^{2}}$. The quantum numbers corresponding to the outgoing lepton state are taken to be $\breve{q}=\left(n, q^{2}, q^{3}\right)$, together with a polarization index $r_{l}$. In this case the energy is given by $E_{l}=\sqrt{m_{l}^{2}+2 n B_{e}+\left(q^{3}\right)^{2}}$ (see Appendix A.3). Finally, being electrically neutral, the outgoing antineutrino is taken to be in a state of momentum $\vec{k}=\left(k^{1}, k^{2}, k^{3}\right)$ and polarization $r_{\bar{\nu}_{l}}$, with energy $E_{\bar{\nu}_{l}}=\sqrt{\left(k^{1}\right)^{2}+\left(k^{2}\right)^{2}+\left(k^{3}\right)^{2}}=|\vec{k}|$.
On general grounds, the decay width for the process is given by

$$
\begin{aligned}
\Gamma_{l}^{-}(B)= & \lim _{T \rightarrow \infty} \frac{1}{n_{\pi^{-}}} \sum_{r_{l}} \sum_{n=0}^{\infty} \int \frac{d q^{2} d q^{3}}{(2 \pi)^{3} 2 E_{l}} \\
& \times \sum_{r_{\bar{\nu}_{l}}} \int \frac{d^{3} k}{(2 \pi)^{3} 2 E_{\bar{\nu}_{l}}} \frac{\left|(\mathcal{S}-1)_{f i}\right|^{2}}{T},
\end{aligned}
$$

where $(\mathcal{S}-1)_{f i}$ is the relevant $\mathcal{S}$-matrix element between the initial and final states, and the particle number $n_{\pi^{-}}$ associated with the initial $\pi^{-}$state is given in Eq. (A17). Thus, one has

$$
\begin{aligned}
\Gamma_{l}^{-}(B)= & \lim _{S, T \rightarrow \infty} \frac{1}{2 E_{\pi^{-}}} \sum_{n=0}^{\infty} \int \frac{d q^{2} d q^{3}}{(2 \pi)^{3} 2 E_{l}} \frac{d^{3} k}{(2 \pi)^{3} 2 E_{\bar{\nu}_{l}}} \\
& \times \sum_{r_{l}, r_{\bar{\nu}_{l}}} \frac{\left|\left\langle l\left(\breve{q}, r_{l}\right) \bar{\nu}_{l}\left(\vec{k}, r_{\bar{\nu}_{l}}\right)\left|\mathcal{L}_{W}\right| \pi^{-}(\breve{p})\right\rangle\right|^{2}}{2 \pi S T},
\end{aligned}
$$

where $\mathcal{L}_{W}$ is the usual $V-A$ four-fermion effective weak interaction Langrangian integrated over space-time, while $T$ and $S$ are the time interval and the surface on the $x^{2} x^{3}$ plane in which the interaction is active. At the end of the calculation, the limit $S, T \rightarrow \infty$ will be taken. Let us recall that, according to our gauge choice, the motion in the $x^{1}$ axis is bounded. Using the notation introduced in Appendix A, the matrix element in Eq. (32) is given by

$$
\left\langle l\left(\breve{q}, r_{l}\right) \bar{\nu}_{l}\left(\vec{k}, r_{\bar{\nu}_{l}}\right)\left|\mathcal{L}_{W}\right| \pi^{-}(\breve{p})\right\rangle=-\frac{G_{F}}{\sqrt{2}} \cos \theta_{c} \int d^{4} x H_{L}^{-, \mu}(x, \breve{p}) \bar{U}_{l}^{-}\left(x, \breve{q}, r_{l}\right) \gamma_{\mu}\left(1-\gamma_{5}\right) V_{\nu_{l}}\left(x, \vec{k}, r_{\bar{\nu}_{l}}\right),
$$

where $H_{L}^{-, \mu}(x, \breve{p})$ stands for the matrix element of the hadronic current [see Eqs. (19) and (20)],

$$
H_{L}^{-, \mu}(x, \breve{p})=H_{V}^{-, \mu}(x, \breve{p})-H_{A}^{-, \mu}(x, \breve{p})=\left\langle 0\left|\bar{\psi}_{u}(x) \gamma^{\mu}\left(1-\gamma_{5}\right) \psi_{d}(x)\right| \pi^{-}(\breve{p})\right\rangle
$$

Thus, from Eqs. (A6), (A20) and (A25), the decay amplitude is given by

$$
\left\langle l\left(\breve{q}, r_{l}\right) \bar{\nu}_{l}\left(\vec{k}, r_{\bar{\nu}_{l}}\right)\left|\mathcal{L}_{W}\right| \pi^{-}(\breve{p})\right\rangle=-\frac{G_{F}}{\sqrt{2}} \cos \theta_{c} \sum_{\lambda= \pm} \bar{u}_{l}^{-}\left(\breve{q}, r_{l}\right) \Delta^{\lambda} \gamma_{\mu}\left(1-\gamma_{5}\right) v_{\nu_{l}}\left(\vec{k}, r_{\bar{\nu}_{l}}\right) \int d^{4} x H_{L}^{-, \mu}(x, \breve{p}) E_{\bar{q}, \lambda}^{-}(x)^{*} e^{i k x}
$$

where $k^{\mu}=\left(E_{\bar{l}_{l}}, \vec{k}\right)$ and $\bar{q}=\left(E_{l}, \breve{q}\right)$. Given our gauge choice for the external magnetic field, the electromagnetic potential depends only on the $x^{1}$ coordinate. Consequently, the momentum is conserved in the 0,2 and 3 directions, leading to the corresponding delta functions when the space-time integration in Eq. (35) is performed. We obtain

$$
\int d^{4} x H_{L}^{-, \mu}(x, \breve{p}) E_{\bar{q}, \lambda}^{-}(x)^{*} e^{i k x}=(2 \pi)^{3} \delta\left(E_{\pi^{-}}-E_{l}-E_{\bar{\nu}_{l}}\right) \prod_{i=2,3} \delta\left(p^{i}-q^{i}-k^{i}\right) M_{\lambda}^{-, \mu},
$$


where the $M_{\lambda}^{-, \mu}$ functions will be explicitly given below. In this way, the amplitude can be written as

$$
\left\langle l\left(\breve{q}, r_{l}\right) \bar{\nu}_{l}\left(\vec{k}, r_{\bar{\nu}_{l}}\right)\left|\mathcal{L}_{W}\right| \pi^{-}(\breve{p})\right\rangle=(2 \pi)^{3} \delta\left(E_{\pi^{-}}-E_{l}-E_{\bar{\nu}_{l}}\right) \prod_{i=2,3} \delta\left(p^{i}-q^{i}-k^{i}\right) \mathcal{M}_{\pi^{-} \rightarrow l \bar{\nu}_{l}},
$$

where

$$
\mathcal{M}_{\pi^{-} \rightarrow l \bar{\nu}_{l}}=-\frac{G_{F}}{\sqrt{2}} \cos \theta_{c} \sum_{\lambda= \pm} \bar{u}_{l}^{-}\left(\breve{q}, r_{l}\right) \Delta^{\lambda} \gamma_{\mu}\left(1-\gamma_{5}\right) v_{\nu_{l}}\left(\vec{k}, r_{\bar{\nu}_{l}}\right) M_{\lambda}^{-, \mu}
$$

Thus, replacing in Eq. (32) and taking the limit of infinite space-time volume, the decay width is given by

$$
\Gamma_{l}^{-}(B)=\frac{1}{4 \pi E_{\pi^{-}}} \sum_{n=0}^{\infty} \int \frac{d q^{2} d q^{3}}{2 E_{l}} \frac{d^{3} k}{2 E_{\bar{\nu}_{l}}} \frac{1}{(2 \pi)^{3}} \delta\left(E_{\pi^{-}}-E_{l}-E_{\bar{\nu}_{l}}\right) \prod_{i=2,3} \delta\left(p^{i}-q^{i}-k^{i}\right) \overline{\left|\mathcal{M}_{\pi^{-} \rightarrow l \bar{\nu}_{l}}\right|^{2}}
$$

where

$$
\overline{\left|\mathcal{M}_{\pi^{-} \rightarrow l \bar{\nu}_{l}}\right|^{2}} \equiv \sum_{r_{l}, r_{\bar{\nu}_{l}}}\left|\mathcal{M}_{\pi^{-} \rightarrow l \bar{\nu}_{l}}\right|^{2}
$$

As customary, when putting the expression on the rhs of Eq. (37) into Eq. (32) we have replaced $\left[\delta\left(E_{\pi^{-}}-E_{l}-E_{\bar{\nu}_{l}}\right)\right]^{2}$ by $\lim _{T \rightarrow \infty} T \delta\left(E_{\pi^{-}}-E_{l}-E_{\bar{\nu}_{l}}\right)$ and $\left[\Pi_{i=2,3} \delta\left(p^{i}-q^{i}-k^{i}\right)\right]^{2}$ by $\lim _{S \rightarrow \infty} S \Pi_{i=2,3} \delta\left(p^{i}-q^{i}-k^{i}\right)$.

Now, as it is usually done, we concentrate on the situation in which the decaying pion is in the state of lowest energy. This corresponds to the case $\ell=0$ and $p^{3}=0$. Moreover, as will be shown below, neither the pion energy nor the decay width depend on the value of $p^{2}$. The expression for the decay width can be worked out, leading to

$$
\begin{aligned}
\Gamma_{l}^{-}(B)= & \frac{1}{16 \pi E_{\pi^{-}}^{2}} \sum_{n=0}^{n_{\max }} \int \frac{d^{2} k_{\perp}}{(2 \pi)^{2}} \int \frac{d k^{3}}{2 \pi} \frac{1}{\overline{k^{3}}} \\
& \times\left[\delta\left(k^{3}-\overline{k^{3}}\right)+\delta\left(k^{3}+\overline{k^{3}}\right)\right] \overline{\left|\mathcal{M}_{\pi^{-} \rightarrow l \bar{\nu}_{l}}\right|^{2}}
\end{aligned}
$$

where we have used the definitions

$$
\begin{gathered}
n_{\max }=\frac{m_{\pi^{-}}^{2}-m_{l}^{2}+B_{e}}{2 B_{e}} \\
\vec{k}_{\perp}=\left(k^{1}, k^{2}\right)
\end{gathered}
$$

$\overline{k^{3}}=\frac{\sqrt{E_{\pi^{-}}^{4}-2 E_{\pi^{-}}^{2}\left(m_{l}^{2}+2 n B_{e}+k_{\perp}^{2}\right)+\left(m_{l}^{2}+2 n B_{e}-k_{\perp}^{2}\right)^{2}}}{2 E_{\pi^{-}}}$,

and the integral over $k_{\perp}$ space is restricted to $\left|\vec{k}_{\perp}\right| \leq E_{\pi^{-}}-\sqrt{m_{l}^{2}+2 n B_{e}}$. In addition, in the amplitude $\mathcal{M}_{\pi^{-} \rightarrow l \bar{\nu}_{l}}$ one should take $q^{3}=-k^{3}$.

\section{B. Evaluation of the weak decay amplitude}

As a second step we concentrate on the evaluation of the sum of the squared amplitudes for all possible lepton polarizations, $\overline{\left|\mathcal{M}_{\pi^{-} \rightarrow l \bar{\nu}_{l}}\right|^{2}}$. Using the properties of electron and neutrino spinors in Eqs. (A7) and (A23), a somewhat tedious but straightforward calculation leads to

$$
\begin{aligned}
\overline{\left|\mathcal{M}_{\pi^{-} \rightarrow l \bar{\nu}_{l}}\right|^{2}}= & 2 G_{F}^{2} \cos ^{2} \theta_{c}\left\{\left(E_{l}-q^{3}\right)\left[\left(E_{\bar{\nu}_{l}}-k^{3}\right)\left|M_{\|,+}^{-,+}\right|^{2}+\left(E_{\bar{\nu}_{l}}+k^{3}\right)\left|M_{\perp,+,}^{-,-}\right|^{2}-2 \operatorname{Re}\left(\left(M_{\|,+}^{-,+}\right)^{*} M_{\perp,+}^{-,-} k^{+}\right)\right]\right. \\
& +\left(E_{l}+q^{3}\right)\left[\left(E_{\bar{\nu}_{l}}+k^{3}\right)\left|M_{\|,-,}^{-,-}\right|^{2}+\left(E_{\bar{\nu}_{l}}-k^{3}\right)\left|M_{\perp,-}^{-,+}\right|^{2}-2 \operatorname{Re}\left(\left(M_{\|,-}^{-,-}\right)^{*} M_{\perp,-,}^{-,+} k^{-}\right)\right] \\
& +2 \sqrt{2 n B_{e}}\left[\left(E_{\bar{\nu}_{l}}+k^{3}\right) \operatorname{Im}\left(\left(M_{\|,-}^{-,-}\right)^{*} M_{\perp,+}^{-,-}\right)-\left(E_{\bar{\nu}_{l}}-k^{3}\right) \operatorname{Im}\left(\left(M_{\|,+}^{-,+}\right)^{*} M_{\perp,-}^{-,+}\right)\right. \\
& \left.\left.-\operatorname{Im}\left(M_{\|,+}^{-,+}\left(M_{\|,-}^{-,-}\right)^{*} k^{-}\right)-\operatorname{Im}\left(M_{\perp_{,}+}^{-,-}\left(M_{\perp,-}^{-,+}\right)^{*} k^{+}\right)\right]\right\},
\end{aligned}
$$

where we have used the definitions

$$
M_{\|, \lambda}^{-, \epsilon}=M_{\lambda}^{-, 0}+\epsilon M_{\lambda}^{-, 3}, \quad M_{\perp, \lambda}^{-, \epsilon}=M_{\lambda}^{-, 1}+i \epsilon M_{\lambda}^{-, 2}, \quad k^{\epsilon}=k^{1}+i \epsilon k^{2} .
$$

To proceed we need to calculate $M_{\|, \lambda}^{-, \epsilon}$ and $M_{\perp, \lambda}^{-, \epsilon}$, which are related to the matrix elements of the hadronic current through Eq. (36). Defining 


$$
\begin{aligned}
& H_{\|, L}^{-, \epsilon}=H_{L}^{-, 0}+\epsilon H_{L}^{-, 3}=H_{\|, V}^{-, \epsilon}-H_{\|, A}^{-, \epsilon}, \\
& H_{\perp, L}^{-, \epsilon}=H_{L}^{-, 1}+i \epsilon H_{L}^{-, 2}=H_{\perp, V}^{-, \epsilon}-H_{\perp, A}^{-, \epsilon},
\end{aligned}
$$

from the expressions in Eqs. (27)-(30) we get

$$
\begin{aligned}
& H_{\|, L}^{-, \epsilon}(x, \bar{p})=i \sqrt{2}\left(f_{\pi^{-}}^{(A 1)}-\epsilon f_{\pi^{-}}^{(V)}\right)\left(E_{\pi^{-}}+\epsilon p^{3}\right) \mathbb{F}_{\bar{p}}^{-}(x), \\
& H_{\perp, L}^{-, \epsilon}(x, \bar{p})=-\epsilon \sqrt{2}\left(f_{\pi^{-}}^{(A 1)}+\epsilon f_{\pi^{-}}^{(A 2)}-f_{\pi^{-}}^{(A 3)}\right) \sqrt{B_{e}(2 \ell+1+\epsilon)} \mathbb{F}_{\bar{p}+\epsilon}^{-}(x) .
\end{aligned}
$$

Now the integral over space-time variables in Eq. (36) can be carried out as described in Appendix C. In this way one gets

$$
\begin{aligned}
& M_{\|, \lambda}^{-, \epsilon}=i \sqrt{2}\left(f_{\pi^{-}}^{(A 1)}-\epsilon f_{\pi^{-}}^{(V)}\right)\left(E_{\pi^{-}}+\epsilon p^{3}\right) \mathcal{G}_{\ell, n}^{-, \lambda}\left(k_{\perp}\right) e^{i k^{1}\left(p^{2}+q^{2}\right) /\left(2 B_{e}\right)} \\
& M_{\perp, \lambda}^{-, \epsilon}=-\epsilon \sqrt{2}\left(f_{\pi^{-}}^{(A 1)}+\epsilon f_{\pi^{-}}^{(A 2)}-f_{\pi^{-}}^{(A 3)}\right) \sqrt{B_{e}(2 \ell+1+\epsilon)} \mathcal{G}_{\ell+\epsilon, n}^{-, \lambda}\left(k_{\perp}\right) e^{i k^{1}\left(p^{2}+q^{2}\right) /\left(2 B_{e}\right)},
\end{aligned}
$$

where the functions $\mathcal{G}_{\ell, n}^{-, \lambda}\left(k_{\perp}\right)$ are explicitly given in Eq. (C9). Notice that $M_{\|, \lambda}^{,, \epsilon}$ and $M_{\perp, \lambda}^{-, \epsilon}$ depend on $p^{2}$ and $q^{2}$ only through a global common phase; therefore the decay width turns out to be $p^{2}$ and $q^{2}$ independent.

Let us consider once again the case in which the decaying pion is in the state of lowest energy. Taking $\ell=0$, from Eq. (C9) one gets

$$
\begin{aligned}
\mathcal{G}_{-1, n}^{-, \pm}\left(k_{\perp}\right) & =0, \\
\mathcal{G}_{0, n}^{-,-}\left(k_{\perp}\right) & =2 \pi \exp \left(-\frac{k_{\perp}^{2}}{4 B_{e}}\right) \frac{1}{\sqrt{n !}}\left(\frac{-i k^{-}}{\sqrt{2 B_{e}}}\right)^{n}, \\
\mathcal{G}_{0, n}^{-,+}\left(k_{\perp}\right) & =\left(1-\delta_{n 0}\right) \mathcal{G}_{0, n-1}^{-,-}\left(k_{\perp}\right), \\
\mathcal{G}_{1, n}^{-,-}\left(k_{\perp}\right) & =2 \pi \exp \left(-\frac{k_{\perp}^{2}}{4 B_{e}}\right) \frac{1}{\sqrt{n !}}\left(\frac{-i k^{-}}{\sqrt{2 B_{e}}}\right)^{n-1}\left(n-\frac{k_{\perp}^{2}}{2 B_{e}}\right), \\
\mathcal{G}_{1, n}^{-,+}\left(k_{\perp}\right) & =\left(1-\delta_{n 0}\right) \mathcal{G}_{1, n-1}^{-,-}\left(k_{\perp}\right) .
\end{aligned}
$$

The result obtained for the sum of the squared amplitudes, taking $\ell=0$ and $p^{3}=0$, can be replaced in the expression for the partial decay width $\Gamma_{l}^{-}(B)$, Eq. (41). Notice that for a pion in the lowest energy state the "perpendicular" amplitudes $M_{\perp, \lambda}^{-, \epsilon}$ vanish for $\epsilon=-1$. Thus, from Eq. (49), it is seen that the partial decay width will depend on three form factor combinations, namely

$$
\begin{aligned}
& a_{\pi^{-}}=f_{\pi^{-}}^{(A 1)}-f_{\pi^{-}}^{(V)}, \quad b_{\pi^{-}}=f_{\pi^{-}}^{(A 1)}+f_{\pi^{-}}^{(V)}, \\
& c_{\pi^{-}}=f_{\pi^{-}}^{(A 1)}+f_{\pi^{-}}^{(A 2)}-f_{\pi^{-}}^{(A 3)} .
\end{aligned}
$$

We recall that the form factors are in general functions of $\ell$, $p^{3}$ and $B$ (to be evaluated in this case at $\ell=0, p^{3}=0$ ). In this way, after some algebra one arrives at

$$
\Gamma_{l}^{-}(B)=\frac{G_{F}^{2} \cos ^{2} \theta_{c}}{2 \pi E_{\pi^{-}}^{2}} B_{e} \sum_{n=0}^{n_{\max }} \int_{0}^{x_{\max }} d x \frac{1}{\overline{k^{3}}} \frac{x^{n-1}}{n !} e^{-x} A_{\pi^{-}}^{(n)}(x),
$$

where we have introduced the dimensionless variable $x=$ $k_{\perp}^{2} /\left(2 B_{e}\right)$ and used the definitions

$$
\begin{aligned}
A_{\pi^{-}}^{(n)}(x)= & {\left[E_{\pi^{-}}^{2}-2 B_{e}(n-x)-m_{l}^{2}\right]\left[\frac{m_{l}^{2}}{2}\left(n\left|a_{\pi^{-}}\right|^{2}+x\left|b_{\pi^{-}}\right|^{2}\right)\right.} \\
& \left.+B_{e}(n-x)\left(n\left|a_{\pi^{-}}-c_{\pi^{-}}\right|^{2}+x\left|b_{\pi^{-}}-c_{\pi^{-}}\right|^{2}\right)\right] \\
& +2 B_{e} x\left[E_{\pi^{-}}^{2}\left(n\left|a_{\pi^{-}}-b_{\pi^{-}}\right|^{2}-(n-x)\left|b_{\pi^{-}}-c_{\pi^{-}}\right|^{2}\right)\right. \\
& \left.+(n-x) m_{l}^{2}\left|c_{\pi^{-}}\right|^{2}\right]
\end{aligned}
$$

and

$$
x_{\max }=\frac{\left(E_{\pi^{-}}-\sqrt{2 n B_{e}+m_{l}^{2}}\right)^{2}}{2 B_{e}} .
$$

In addition, from Eq. (44) one has

$$
\overline{k^{3}}=\frac{1}{2 E_{\pi^{-}}}\left\{\left[E_{\pi^{-}}^{2}-2 B_{e}(n-x)-m_{l}^{2}\right]^{2}-8 B_{e} E_{\pi^{-}}^{2} x\right\}^{1 / 2},
$$

while $n_{\max }$ is given by Eq. (42). Notice that for $\ell=0$, $p^{3}=0$, the $\pi^{-}$energy is given by $E_{\pi^{-}}=\sqrt{B_{e}+m_{\pi^{-}}^{2}}$.

It is worth remarking that the above expression for $\Gamma_{l}^{-}(B)$ corresponds to the case $B>0, s=\sigma=-$, in Eqs. (27)(30). For $B<0, s=-\sigma=+$, we obtain an expression for $\Gamma_{l}^{-}(B)$ similar to that in Eq. (52), where in the function $A_{\pi^{-}}^{(n)}(x)$ one has to exchange $a_{\pi^{-}} \leftrightarrow b_{\pi^{-}}$. Recalling that $f_{\pi^{-}}^{(V)}$ has to be an odd function of $B$, it is seen that the partial decay width is invariant under the exchange $B \rightarrow-B$, as expected.

\section{Large magnetic field limit}

It is interesting to study the case of a large external magnetic field. As stated, since the pion is built with 
charged quarks, the pion mass will depend in general on the magnetic field. Now, if the mass growth is relatively mild, for large magnetic fields one should get $B_{e}>m_{\pi}^{2}-m_{l}^{2}$. In fact, this is what is obtained from lattice QCD calculations [31] as well as from effective approaches like the NambuJona-Lasinio model [26], for values of $B_{e}$ say $\gtrsim 0.05 \mathrm{GeV}^{2}$. According to Eq. (42), this implies $n_{\max }=0$; hence the outgoing muon or electron (assuming that the energy is below the $\tau$ production threshold) has to lie in its lowest Landau level $n=0$. In this case the expression for the partial width simplifies to

$$
\begin{aligned}
\Gamma_{l, 0}^{-}(B)= & \frac{G_{F}^{2} \cos ^{2} \theta_{c}}{4 \pi E_{\pi^{-}}} \int_{0}^{E_{\pi^{-}}-m_{l}} d k_{\perp} k_{\perp} \frac{e^{-k_{\perp}^{2} /\left(2 B_{e}\right)}}{\bar{k}^{3}} \\
& \times\left[m_{l}^{2}\left(E_{\bar{\nu}_{l}}\left|b_{\pi^{-}}\right|^{2}-\frac{k_{\perp}^{2}}{E_{\pi^{-}}}\left|c_{\pi^{-}}\right|^{2}\right)\right. \\
& \left.+E_{l} k_{\perp}^{2}\left|b_{\pi^{-}}-c_{\pi^{-}}\right|^{2}\right],
\end{aligned}
$$

where $E_{\bar{\nu}_{l}}=E_{\pi^{-}}-E_{l}=\sqrt{\bar{k}^{3^{2}}+k_{\perp}^{2}}$ and $E_{l}=\sqrt{\bar{k}^{3^{2}}+m_{l}^{2}}$, with

$$
\overline{k^{3}}=\frac{1}{2 E_{\pi^{-}}}\left[\left(E_{\pi^{-}}^{2}+k_{\perp}^{2}-m_{l}^{2}\right)^{2}-4 E_{\pi^{-}}^{2} k_{\perp}^{2}\right]^{1 / 2} .
$$

A further simplification can be obtained in the case where the squared lepton mass can be neglected in comparison with $B_{e}$ (or, equivalently, in comparison with $E_{\pi^{-}}^{2}$, which is expected to grow approximately as $B_{e}$ ). Setting $m_{l}=0$, one has $E_{l}=\bar{k}^{3}$, and the integral over $k_{\perp}$ extends up to $E_{\pi^{-}}$. Thus, the decay width is given by

$$
\begin{aligned}
\left.\Gamma_{l, 0}^{-}(B)\right|_{m_{l}=0}= & \frac{G_{F}^{2} \cos ^{2} \theta_{c}}{2 \pi E_{\pi^{-}}} \int_{0}^{E_{\pi^{-}}} d k_{\perp} k_{\perp}^{3} e^{-k_{\perp}^{2} /\left(2 B_{e}\right)}\left|b_{\pi^{-}}-c_{\pi^{-}}\right|^{2} \\
= & \frac{G_{F}^{2} \cos ^{2} \theta_{c}}{\pi} \frac{B_{e}^{2}}{E_{\pi^{-}}}\left[1-\left(1+\frac{E_{\pi^{-}}^{2}}{2 B_{e}}\right) e^{-E_{\pi^{-}}^{2} /\left(2 B_{e}\right)}\right] \\
& \times\left|b_{\pi^{-}}-c_{\pi^{-}}\right|^{2}
\end{aligned}
$$

where, according to the definitions in Eq. (51),

$$
b_{\pi^{-}}-c_{\pi^{-}}=f_{\pi^{-}}^{(V)}-f_{\pi^{-}}^{(A 2)}+f_{\pi^{-}}^{(A 3)} .
$$

It is worth stressing that the decay width does not vanish in the limit $m_{l}=0$; i.e., it does not show the helicity suppression found in the $B=0$ case. In fact, it turns out to grow with the magnetic field as $B_{e}^{2} / E_{\pi^{-}}$, with some suppression due to the factor in square brackets. Moreover, it is seen that the contributions of the usual pion decay form factor, $f_{\pi^{-}}^{(A 1)}$, get canceled in Eq. (59); hence the decay width is proportional to a combination of form factors that do not contribute to the hadronic amplitude in the absence of the external magnetic field. Clearly, the relevance of Eq. (58) depends on whether these form factors are non-negligible for magnetic fields that are much larger than the lepton mass squared. While this is likely to happen for the $\pi^{-}$decay to $e \bar{\nu}_{e}$, in the case of the muon (and of course, the tau) the situation is less clear, and the corrections arising from a finite lepton mass should be taken into account. Interestingly, it is possible to obtain relatively simple expressions for the $\pi^{-} \rightarrow l \bar{\nu}_{l}$ decay width at leading order in the ratio $m_{l} / E_{\pi^{-}}$. From Eq. (56) one gets

$$
\begin{aligned}
\Gamma_{l, 0}^{-}(B)= & \left.\Gamma_{l, 0}^{-}(B)\right|_{m_{l}=0}+\frac{G_{F}^{2} \cos ^{2} \theta_{c}}{2 \pi} \frac{B_{e} e^{-E_{\pi^{-}}^{2} /\left(2 B_{e}\right)}}{E_{\pi^{-}}} \\
& \times\left[f_{1}\left|b_{\pi^{-}}\right|^{2}-2 f_{2} \operatorname{Re}\left(b_{\pi^{-}}^{*} c_{\pi^{-}}\right)+f_{3}\left|c_{\pi^{-}}\right|^{2}\right] m_{l}^{2} \\
& +\mathcal{O}\left(\frac{m_{l}^{3}}{E_{\pi^{-}}^{3}}\right),
\end{aligned}
$$

where

$$
\begin{gathered}
f_{1}=(1+\alpha)^{2}-(1+2 \alpha) e^{\alpha}+2 \alpha^{2}\left(I(\alpha)-\ln \frac{m_{l}}{E_{\pi^{-}}}\right), \\
f_{2}=\alpha(2+\alpha)-2 \alpha e^{\alpha}+2 \alpha(\alpha-1)\left(I(\alpha)-\ln \frac{m_{l}}{E_{\pi^{-}}}\right), \\
f_{3}=\alpha^{2}+2 \alpha-2+2(1-\alpha) e^{\alpha} \\
+2 \alpha(\alpha-2)\left(I(\alpha)-\ln \frac{m_{l}}{E_{\pi^{-}}}\right),
\end{gathered}
$$

with $\alpha=E_{\pi^{-}}^{2} / 2 B_{e}$ and $I(\alpha)=\int_{0}^{1} d x\left(e^{\alpha x}-1\right) / x$. It is seen that for $m_{l}=m_{\mu}=105.65 \mathrm{MeV}$ and $B_{e} \gtrsim 0.3 \mathrm{GeV}^{2}$, Eq. (60) approximates the full result in Eq. (56) within $15 \%$ accuracy.

It is also interesting at this point to compare our result in Eq. (56) with the expression quoted in Eq. (5) of Ref. [31], which also corresponds to the limit of a large external magnetic field. The authors of that work make some approximations for the motion of a charged pion in the presence of the magnetic field, concluding that only one of the two possible antineutrino polarizations can contribute to the decay amplitude. Moreover, based on considerations of angular momentum conservation, they assume that the antineutrino momentum in the perpendicular plane $\vec{k}_{\perp}$ vanishes. In fact, it can be seen that if one imposes such a condition in Eq. (56), the result quoted in Ref. [31] can be recovered. However, we find that if one takes full account of the effect of the magnetic field on charged pion wave functions, conservation laws do not imply $\vec{k}_{\perp}=0$; therefore one should integrate over all possible values of the antineutrino momentum, as in Eq. (56). Another main difference between our work and the analysis in Ref. [31] is that our calculations include a perpendicular piece of the hadronic amplitude (related to $c_{\pi^{-}}$), which arises due to the 
presence of a $\pi^{-}$zero point motion in the perpendicular plane, even in the $\ell=0$ state.

The study of angular momentum in the presence of magnetic fields has been addressed in the recent literature (see e.g., Ref. [34]) and deserves some discussion. As suggested in Ref. [34], the consequences of the axial symmetry of the problem, as well as the physical meaning of angular momenta, can be better understood if one works in the symmetric gauge. Having this in mind, we have rederived the $\pi^{-} \rightarrow l \bar{\nu}_{l}$ decay width in this gauge, considering for simplicity the case of a charged pion in its lowest energy state and a charged lepton in the lowest Landau level. For the spatial wave functions of the pion and charged lepton states we have used the functions given e.g., in Eq. (17) of Ref. [34] (see also Ref. [35]). These functions are eigenstates of the $z$ component of the orbital angular momentum operator, $L^{3}$, with eigenvalues say $M_{p}$ and $M_{q}$ for the pion and the charged lepton, respectively. If the particles are in the lowest Landau level, it is found that $M_{p}$ and $M_{q}$ can take any integer value $\leq 0$. In order to deal with right-handed antineutrino states we have used spinors that are eigenstates of the $z$ component of the total angular momentum, $J^{3}$, with eigenvalue $j^{3}$. Replacing in the corresponding weak decay matrix element and performing the integral over space-time coordinates we obtain

$$
\begin{aligned}
& \left\langle l\left(q^{3}, n=0, M_{q}\right) \bar{\nu}_{l}\left(k^{3}, k_{\perp}, j^{3}\right)\left|\mathcal{L}_{W}\right| \pi^{-}\left(p^{3}, \ell=0, M_{p}\right)\right\rangle \\
& =(2 \pi)^{3} \delta\left(E_{\pi^{-}}-E_{l}-E_{\bar{\nu}_{l}}\right) \delta\left(p^{3}-q^{3}-k^{3}\right) \delta_{M_{p}, M_{q}-\frac{1}{2}+j^{3}} \mathcal{M},
\end{aligned}
$$

where $\mathcal{M}$ is a function that depends on the pion decay form factors $f_{\pi^{-}}^{\left(V, A_{i}\right)}$ and on particle quantum numbers. Hence, in this gauge it is clearly seen that angular momentum conservation leads to the selection rule $M_{p}=$ $M_{q}-1 / 2+j^{3}$, without requiring $k_{\perp}=\left|\vec{k}_{\perp}\right|=0$. Using the explicit form of $\mathcal{M}$ and taking $p^{3}=0$, we have then calculated the sum/integral over all allowed outgoing states. The outcome (which, as expected, does not depend on $M_{p}$ ), leads to an expression for the decay width that coincides exactly with the one quoted in Eq. (56), confirming the gauge independence of our result. It is also worth mentioning that, in addition to the usual "canonical" angular momentum, one can define "mechanical" angular momenta replacing particle momenta $P^{\mu}$ by $P_{\text {mech }}^{\mu} \equiv P^{\mu}-Q \mathcal{A}^{\mu}$ (a detailed comparison of both quantities for various gauge choices is given in Ref. [34]). Interestingly, for the $\pi^{-} \rightarrow l \bar{\nu}_{l}$ decay in the limit of large $B$ (when only the $n=0$ state contributes) it is found that the third components of mechanical angular momenta of incoming and outgoing states only coincide when the transverse antineutrino momentum vanishes, i.e., for $\left|k_{\perp}\right|=0$.

\section{D. $B \rightarrow 0$ limit}

In the $B \rightarrow 0$ limit our expressions should reduce to the well-known result for the $\pi^{-} \rightarrow l \bar{\nu}_{l}$ decay width obtained in the absence of external fields. For simplicity we consider the decay of a $\pi^{-}$in its fundamental state, taking $\ell=0$ and $p^{2}=p^{3}=0$. Then, in the $B \rightarrow 0$ limit the decay width should reduce to that of a $\pi^{-}$at rest, namely

$$
\begin{aligned}
\Gamma_{l}^{-}(0)= & \frac{1}{2 m_{\pi}} \int \frac{d^{3} k}{(2 \pi)^{3} 2 E_{\bar{\nu}_{l}}} \frac{d^{3} q}{(2 \pi)^{3} 2 E_{l}}(2 \pi)^{4} \delta^{(4)}(p-q-k) \\
& \times\left(G_{F} \cos \theta_{c} f_{\pi}\right)^{2} 8 m_{\pi}^{2}\left(E_{l} E_{\bar{\nu}_{l}}+\vec{q} \cdot \vec{k}\right) \\
= & \frac{\left(G_{F} \cos \theta_{c} f_{\pi}\right)^{2}}{4 \pi} m_{\pi} m_{l}^{2}\left(1-\frac{m_{l}^{2}}{m_{\pi}^{2}}\right)^{2}
\end{aligned}
$$

where $p^{\mu}=\left(m_{\pi}, \overrightarrow{0}\right) ; q^{\mu}=\left(E_{l}, \vec{q}\right)$; and $k^{\mu}=\left(E_{\bar{\nu}_{l}}, \vec{k}\right)$ stand for the pion, lepton and antineutrino four-momenta, respectively. It can be checked that for a given value of the lepton mass the numerical results for Eqs. (66) and (52), in the limit $\quad B_{e} \rightarrow 0, \quad n_{\max } \rightarrow \infty, \quad a_{\pi^{-}}=b_{\pi^{-}}=c_{\pi^{-}}=f_{\pi}$, are coincident. However, the comparison between Eqs. (39) and (65) still leads to the question of how one can arrive at four-momentum conservation in the limit of vanishing external magnetic field. Hence, the goal of this section is to recover analytically the Dirac deltas of momentum conservation, obtaining Eq. (65) from Eq. (39) in the $B \rightarrow 0$ limit.

The presence of a magnetic field implies the existence of a characteristic time and a characteristic length of the system, given in natural units by $B_{e}^{-1 / 2}$, which is usually called the "magnetic length." In the $B \rightarrow 0$ limit these have to be much larger than the time $T$ and the distance $2 L$ along which the interaction is active, i.e.,

$$
\sqrt{B_{e}} L \ll 1, \quad \sqrt{B_{e}} T \ll 1 .
$$

In addition, we assume that $T$ and $L$ are large enough so that the product of any momentum of the system times $L$ or any energy of the system times $T$ turns out to be much larger than 1 . In particular,

$\sqrt{n B_{e}} L \gg 1, \quad k_{x} L \gg 1, \quad E_{l} T \gg 1, \quad E_{\bar{\nu}} T \gg 1$.

We recall that the lepton energy is given by $E_{l}=$ $\sqrt{m_{l}^{2}+2 n B_{e}+\left(q^{3}\right)^{2}}$, which implies that the magnetic field will contribute significantly to the energy only for very large values of $n$. In fact, from this expression we can infer that the term $2 n B_{e}$ will lead to the $\left(q_{\perp}\right)^{2}$ contribution to the energy in the $B \rightarrow 0$ limit. As $B$ decreases, the contributing leptonic states will have increasingly larger values of $n$, in such a way that $n B$ remains finite. 
From Eqs. (8), (19) and (20), we observe that in the $B \rightarrow 0$ limit the only nonvanishing pion decay form factor is $f_{\pi^{-}}^{(A 1)}$. Thus, from Eqs. (45)-(48) we have

$$
\begin{aligned}
\left|\overline{\left|\mathcal{M}_{\pi^{-} \rightarrow l \bar{\nu}_{l}}\right|^{2}}\right|_{\ell=p^{3}=0}= & 4 G_{F}^{2} \cos ^{2} \theta_{c}\left|f_{\pi^{-}}^{(A 1)}\right|^{2}\left\{E_{\pi^{-}}^{2}\left(E_{l}-q^{3}\right)\left(E_{\bar{\nu}_{l}}-k^{3}\right)\left|\mathcal{I}_{0, n}^{-,+}\right|^{2}+E_{\pi^{-}}^{2}\left(E_{l}+q^{3}\right)\left(E_{\bar{\nu}_{l}}+k^{3}\right)\left|\mathcal{I}_{0, n}^{-,-}\right|^{2}\right. \\
& +2 B_{e}\left(E_{l}+q^{3}\right)\left(E_{\bar{\nu}_{l}}-k^{3}\right)\left|\mathcal{I}_{1, n}^{-,-}\right|^{2}-2 \sqrt{2 B_{e}} E_{\pi^{-}}\left(E_{l}+q^{3}\right) \operatorname{Im}\left(\left(\mathcal{I}_{0, n}^{-,-}\right)^{*} \mathcal{I}_{1, n}^{-,-} k^{-}\right) \\
& \left.+2 \sqrt{2 n B_{e}} E_{\pi^{-}}\left[\sqrt{2 B_{e}}\left(E_{\bar{\nu}_{l}}-k^{3}\right) \operatorname{Im}\left(\left(\mathcal{I}_{0, n}^{-,+}\right)^{*} \mathcal{I}_{1, n}^{-,-}\right)-E_{\pi^{-}} \operatorname{Im}\left(\mathcal{I}_{0, n}^{-,+}\left(\mathcal{I}_{0, n}^{-,-}\right)^{*} k^{-}\right)\right]\right\},
\end{aligned}
$$

where the functions $\mathcal{I}_{\ell, n}^{-, \pm}$are given by

$$
\mathcal{I}_{\ell, n}^{-, \pm}=N_{\ell} N_{n_{\mp}} \int_{-L}^{L} d x^{1} e^{i k^{1} x^{1}} D_{\ell}\left(\sqrt{2 B_{e}} x^{1}\right) D_{n_{\mp}}\left(\sqrt{2 B_{e}} x^{1}+\sqrt{\frac{2}{B_{e}}} q^{2}\right) .
$$

In fact, the latter correspond to the functions $\mathcal{I}_{\ell, n}^{-, \lambda}\left(k_{\perp}, p^{2}, q^{2}\right)$ defined in Eq. (C3), taking $p^{2}=0$ and restricting the integral over $x^{1}$ to the interval $(-L, L)$ in order to take into account the conditions in Eqs. (67) and (68).

Owing to the restriction of the integration interval in Eq. (70), in the $B \rightarrow 0$ limit one has $\sqrt{2 B_{e}} x^{1} \rightarrow 0$. Thus, the factors $N_{\ell} D_{\ell}\left(\sqrt{2 B_{e}} x^{1}\right)$, where $\ell=0$ or 1 , satisfy

$$
\begin{gathered}
N_{0} D_{0}\left(\sqrt{2 B_{e}} x^{1}\right) \underset{\sqrt{2 B_{e}} x^{1} \rightarrow 0}{\rightarrow}\left(4 \pi B_{e}\right)^{1 / 4}, \\
N_{1} D_{1}\left(\sqrt{2 B_{e}} x^{1}\right) \underset{\sqrt{2 B_{e}} x^{1} \rightarrow 0}{\rightarrow}\left(4 \pi B_{e}\right)^{1 / 4} \frac{1}{2} \sqrt{2 B_{e}} x^{1} \sim 0,
\end{gathered}
$$

and the terms with $\mathcal{I}_{1, n}^{-,-}$in Eq. (69) can be neglected. We obtain

$$
\begin{aligned}
\left.\overline{\left|\mathcal{M}_{\pi^{-} \rightarrow \mid \bar{\nu}_{l}}\right|^{2}}\right|_{\ell=p^{3}=0}= & 4 G_{F}^{2} \cos ^{2} \theta_{c}\left|f_{\pi^{-}}^{(A 1)}\right|^{2} E_{\pi^{-}}^{2}\left\{\left(E_{l}-q^{3}\right)\left(E_{\bar{\nu}_{l}}-k^{3}\right)\left|\mathcal{I}_{0, n}^{-,+}\right|^{2}\right. \\
& \left.+\left(E_{l}+q^{3}\right)\left(E_{\bar{\nu}_{l}}+k^{3}\right)\left|\mathcal{I}_{0, n}^{-,-}\right|^{2}-2 \sqrt{2 n B_{e}} \operatorname{Im}\left(\mathcal{I}_{0, n}^{-,+}\left(\mathcal{I}_{0, n}^{-,-}\right)^{*} k^{-}\right)\right\} .
\end{aligned}
$$

The detailed calculation of the functions $\mathcal{I}_{0, n}^{-, \lambda}$ and their contributions to the expression in Eq. (73) is given in Appendix D. We finally arrive at

$$
\begin{aligned}
\left|\overline{\left.\mathcal{M}_{\pi^{-} \rightarrow l \bar{\nu}_{l}}\right|^{2}}\right|_{\ell=p^{3}=0}= & 8 G_{F}^{2} \cos ^{2} \theta_{c}\left|f_{\pi^{-}}^{(A 1)}\right|^{2} 4 \pi L\left(4 \pi B_{e}\right)^{1 / 2} \theta\left(1-\frac{\left|q^{2}\right|}{\sqrt{2 n B_{e}}}\right) \frac{B_{e}}{\bar{q}_{n}} \\
& \times E_{\pi^{-}}^{2}\left\{\left(E_{l} E_{\bar{\nu}_{l}}+q^{2} k^{2}+q^{3} k^{3}\right)\left[\delta\left(k^{1}-\bar{q}_{n}\right)+\delta\left(k^{1}+\bar{q}_{n}\right)\right]\right. \\
& \left.+k^{1} \bar{q}_{n} \delta\left(k^{1}+\bar{q}_{n}\right)-k^{1} \bar{q}_{n} \delta\left(k^{1}-\bar{q}_{n}\right)\right\},
\end{aligned}
$$

where $\bar{q}_{n}=\sqrt{2 n B_{e}-\left(q^{2}\right)^{2}}$. We recall that $n$ has to be a large number, in such a way that $n B_{e}$ is kept finite for small $B$.

We introduce now a new variable $q^{1}$, in the following way:

$$
\begin{aligned}
\left|\overline{\left.\mathcal{M}_{\pi^{-} \rightarrow l \bar{\nu}_{l}}\right|^{2}}\right|_{\ell=p^{3}=0}= & 8 G_{F}^{2} \cos ^{2} \theta_{c}\left|f_{\pi^{-}}^{(A 1)}\right|^{2} 2 L\left(4 \pi B_{e}\right)^{1 / 2} \theta\left(1-\frac{\left|q^{2}\right|}{\sqrt{2 n B_{e}}}\right) \frac{B_{e}}{\bar{q}_{n}} \\
& \times \int d q^{1} 2 \pi \delta\left(q^{1}+k^{1}\right)\left[\delta\left(q^{1}-\bar{q}_{n}\right)+\delta\left(q^{1}+\bar{q}_{n}\right)\right] \\
& \times E_{\pi^{-}}^{2}\left(E_{l} E_{\bar{\nu}_{l}}+q^{1} k^{1}+q^{2} k^{2}+q^{3} k^{3}\right) .
\end{aligned}
$$

Next, let us consider the decay width in Eq. (39). We need to treat with some care the pion density $n_{\pi^{-}}$, which appears in the definition of the width in Eq. (31). In fact, for a finite space length $2 L$, taking into account the approximation in Eq. (71), the pion density will be given by

$$
n_{\pi^{-}}=S \int_{-L}^{L} d x^{1} 2 E_{\pi^{-}}\left(4 \pi B_{e}\right)^{1 / 2}=4 L S E_{\pi^{-}}\left(4 \pi B_{e}\right)^{1 / 2}
$$


This result can be understood by writing the pion density in the form $n_{\pi^{-}}=V 2 E_{\pi^{-}}\left(4 \pi B_{e}\right)^{1 / 2}$, where $V$ is the volume in which the interaction occurs. It is seen that Eq. (76) recovers the pion density in the absence of the magnetic field [see Eq. (A3)] times a factor $\left(4 \pi B_{e}\right)^{1 / 2}$. The latter compensates the fact that in the limit of small $B$, according to our normalization of charged pion states, the spacial wave function of a pion in a zero three-momentum state is $N_{0} D_{0}\left(\sqrt{2 B_{e}} x^{1}\right) \rightarrow\left(4 \pi B_{e}\right)^{1 / 4}$, instead of 1. Now, comparing the result in Eq. (76) with the expression $n_{\pi^{-}}=4 \pi S E_{\pi^{-}}$quoted in Eq. (A17), it comes out that the width in the rhs of Eq. (39) has to be modified by a factor $\pi /\left[L\left(4 \pi B_{e}\right)^{1 / 2}\right]$. In addition, in the limit of small $B$ one can change the sum over $n$ in Eq. (39) by an integral over a variable $\varkappa \equiv 2 n B_{e}$. Hence, for $p^{2}=p^{3}=0$ we get

$$
\begin{aligned}
\Gamma_{l}^{-}(B \rightarrow 0)= & \frac{2 G_{F}^{2} \cos ^{2} \theta_{c}}{\pi E_{\pi^{-}}} \frac{1}{2 B_{e}} \int d \varkappa \int \frac{d q^{2} d q^{3}}{(2 \pi)^{3} 2 E_{l}} \int \frac{d^{3} k}{(2 \pi)^{3} 2 E_{\bar{\nu}_{l}}}(2 \pi)^{3} \\
& \times \delta\left(E_{\pi^{-}}-E_{l}-E_{\bar{\nu}_{l}}\right) \prod_{i=2,3} \delta\left(q^{i}+k^{i}\right) \frac{\pi}{L\left(4 \pi B_{e}\right)^{1 / 2}} \\
& \times \int d q^{1}\left|f_{\pi^{-}}^{(A 1)}\right|^{2} 2 \pi \delta\left(q^{1}+k^{1}\right) 2 L\left(4 \pi B_{e}\right)^{1 / 2} \theta\left(1-\frac{\left|q^{2}\right|}{\sqrt{\varkappa}}\right) \frac{B_{e}}{\sqrt{\varkappa-\left(q^{2}\right)^{2}}} \\
& \times\left[\delta\left(q^{1}-\sqrt{\varkappa-\left(q^{2}\right)^{2}}\right)+\delta\left(q^{1}+\sqrt{\varkappa-\left(q^{2}\right)^{2}}\right)\right] \\
& \times E_{\pi^{-}}^{2}\left(E_{l} E_{\bar{\nu}_{l}}+q^{1} k^{1}+q^{2} k^{2}+q^{3} k^{3}\right) .
\end{aligned}
$$

Finally, we can perform the integral over $\varkappa$. The delta functions fix $q^{1}= \pm \sqrt{\varkappa-\left(q^{2}\right)^{2}}$, leading to the expected result $\varkappa=2 n B_{e}=q_{\perp}^{2}$. Identifying $f_{\pi^{-}}^{(A 1)}$ with $f_{\pi}$ in the $B \rightarrow 0$ limit, we arrive at the expression in Eq. (65).

\section{CONCLUSIONS}

In this work we present a general method to parametrize the one-pion-to-vacuum matrix elements of the vector and axial vector hadronic currents in the presence of an external uniform static magnetic field $\vec{B}$. Choosing this field to be orientated along the 3-axis, we show that for the case of the neutral pion the matrix elements of the parallel (0- and 3-) components of the vector current can be expressed in terms of one single real form factor, $f_{\pi^{0}}^{(V)}$, while the perpendicular (1- and 2-) components vanish identically. For the matrix elements of the axial vector current, two real form factors $f_{\pi^{0}}^{(A 1)}$ and $f_{\pi^{0}}^{(A 3)}$ can be defined. Alternatively, the latter can be written in terms of a parallel and a perpendicular form factor, in consistency with the result obtained in Ref. [29]. In the case of the charged pion, the situation is similar in what concerns the vector current. Namely, the matrix elements of the parallel components can be expressed in terms of a real (in general, nonvanishing) form factor $f_{\pi^{\sigma}}^{(V)}$, common to both $\pi^{+}$and $\pi^{-}$, while the perpendicular (i.e., 1 and 2-) components vanish. This is in agreement with the statement made in Ref. [31]. On the other hand, we find that three form factors, $f_{\pi^{ \pm}}^{(A 1)}, f_{\pi^{ \pm}}^{(A 2)}$ and $f_{\pi^{ \pm}}^{(A 3)}$, are in general required to parametrize the matrix elements of the axial vector hadronic current. Once again, the three of them are real, and they are equal for both pion charges. The matrix elements of the charged pions in Eqs. (27)-(30) can be viewed as a proper generalization of the corresponding expressions given in Ref. [31]. We have included here all possible gauge covariant structures, taking fully into account the effect of the magnetic field on the charged pion wave functions. It should be noticed that in the particular case of a charged pion lying in the lowest Landau level, only two combinations of these three form factors contribute to the decay width.

Using the above results we introduce a general, model-independent framework to study the weak decay $\pi^{-} \rightarrow l \bar{\nu}_{l}$ in the presence of an arbitrary large external magnetic field. For the case in which the decaying pion lies in its state of minimum energy (i.e., in the lowest Landau level, with zero momentum along the 3-direction), we obtain an explicit expression for the $\pi^{-} \rightarrow l \bar{\nu}_{l}$ decay width. The limits of this expression for the cases of strong and weak magnetic fields are also studied, checking that in the limit of $B=0$ it reduces to the usual result. It is interesting to note that the expression obtained in the limit of large magnetic field, Eq. (56), is valid in most cases of physical interest. Namely, we estimate its range of validity to be $0.05 \mathrm{GeV}^{2}<e B<m_{\tau}^{2} \approx 3 \mathrm{GeV}^{2}$. It is seen that our result shows some differences with the one given in Ref. [31], also obtained in the limit of large $B$. We understand that the discrepancies arise from some approximations made in Ref. [31] concerning the motion of a charged pion in the presence of the magnetic field. It is also worth noticing that the decay width does not vanish in the limit $m_{l}=0$; i.e., it does not show the helicity suppression found in the absence of the external magnetic field. 
We finally mention that an obvious application of our work would be to study how weak decay rates of charged pions get modified due to the presence of the magnetic field. To reach this goal, however, the behavior of the decay form factors as functions of the magnetic field should be determined. This would require either the use of LQCD simulations, as proposed in Ref. [31], or to rely on some hadronic effective model. We expect to report soon on such a calculation in the framework of Nambu-Jona-Lasinio-like models.

\section{ACKNOWLEDGMENTS}

This work has been supported in part by Consejo Nacional de Investigaciones Científicas y Técnicas (CONICET) and Agencia Nacional de Promoción Científica y Tecnológica (ANPCyT, Argentina), under Grants No. PIP14-492 and No. PICT14-03-0492; by the National University of La Plata (Argentina), Project No. X824; by the Ministerio de Economía y Competitividad (Spain), under Contract No. FPA2016-77177-C2-1-P; by the Centro de Excelencia Severo Ochoa Programme, Grant No. SEV-2014-0398; and by Generalitat Valenciana (Spain), Grant No. PrometeoII/ 2014/066.

\section{APPENDIX A: PION AND FERMION FIELDS IN A CONSTANT MAGNETIC FIELD}

In this appendix we quote expressions for the different fields used in our work, written in terms of creation and annihilation operators. As in the main text, we assume $\vec{B}=B \hat{x}^{3}$ and make use of the Landau gauge, in which $\mathcal{A}^{\mu}=\left(0,0, B x^{1}, 0\right)$.

\section{Neutral pion and neutrino fields}

The expressions for neutral fields do not get changed by the presence of the external magnetic fields. Thus, they can be written in terms of the usual creation and annihilation operators of definite momentum states. Following the conventions given e.g., in Ref. [36], the neutral pion field is given by

$\phi_{\pi^{0}}(x)=\int \frac{d^{3} p}{(2 \pi)^{3} 2 E_{\pi^{0}}}\left[a(\vec{p}) e^{-i p \cdot x}+a^{\dagger}(\vec{p}) e^{i p \cdot x}\right]$,

where $x=(t, \vec{x})$ and $p=\left(E_{\pi^{0}}, \vec{p}\right)$, with $E_{\pi^{0}}=\sqrt{m_{\pi^{0}}^{2}+|\vec{p}|^{2}}$ (it is worth mentioning that, in the presence of an external field, one could also take into account corrections leading to an anisotropic dispersion relation [29]). The operators $a(\vec{p})$ and $a^{\dagger}(\vec{p})$ satisfy the commutation rule

$$
\left[a(\vec{p}), a^{\dagger}\left(\vec{p}^{\prime}\right)\right]=2 E_{\pi^{0}}(2 \pi)^{3} \delta^{(3)}\left(\vec{p}-\vec{p}^{\prime}\right) .
$$

For a finite volume $V$, the particle number $n_{\pi^{0}}$ associated with the momentum eigenstate $\left|\pi^{0}(\vec{p})\right\rangle=a^{\dagger}(\vec{p})|0\rangle$ is given by

$$
n_{\pi^{0}}=\int_{V} d^{3} x\left\langle\pi^{0}(\vec{p})\left|j_{\pi^{0}}^{0}(t, \vec{x})\right| \pi^{0}(\vec{p})\right\rangle=2 E_{\pi^{0}} V,
$$

where

$$
j_{\pi^{0}}^{0}(x)=i\left[\phi_{\pi^{0}}^{\dagger}(x) \partial^{0} \phi_{\pi^{0}}(x)-\partial^{0} \phi_{\pi^{0}}^{\dagger}(x) \phi_{\pi^{0}}(x)\right] .
$$

The neutrino field can be written as

$$
\begin{aligned}
\psi_{\nu_{l}}(x)= & \sum_{r=1,2} \int \frac{d^{3} k}{(2 \pi)^{3} 2 E_{\nu_{l}}}\left[b(\vec{k}, r) U_{\nu_{l}}(x, \vec{k}, r)\right. \\
& \left.+d(\vec{k}, r)^{\dagger} V_{\nu_{l}}(x, \vec{k}, r)\right],
\end{aligned}
$$

where

$U_{\nu_{l}}(x, \vec{k}, r)=u_{\nu_{l}}(\vec{k}, r) e^{-i k \cdot x}, \quad V_{\nu_{l}}(x, \vec{k}, r)=v_{\nu_{l}}(\vec{k}, r) e^{i k \cdot x}$.

Here $k=\left(E_{\nu_{l}}, \vec{k}\right)$, with $E_{\nu_{l}}=|\vec{k}|$, while $u_{\nu_{l}}(\vec{k}, r)$ and $v_{\nu_{l}}(\vec{k}, r)$ are the usual Dirac spinors with polarization states $r=1$ or 2 . They satisfy

$$
\sum_{r=1,2} u_{\nu_{l}}(\vec{k}, r) \bar{u}_{\nu_{l}}(\vec{k}, r)=\sum_{r=1,2} v_{\nu_{l}}(\vec{k}, r) \bar{v}_{\nu_{l}}(\vec{k}, r)=\not k .
$$

Note that we are assuming that neutrinos are massless. The corresponding creation and annihilation operators satisfy the relations

$$
\begin{aligned}
\left\{b(\vec{k}, r), b\left(\vec{k}^{\prime}, r^{\prime}\right)^{\dagger}\right\} & =\left\{d(\vec{k}, r), d\left(\vec{k}^{\prime}, r^{\prime}\right)^{\dagger}\right\} \\
& =2 E_{\nu_{l}}(2 \pi)^{3} \delta_{r r^{\prime}} \delta^{(3)}\left(\vec{k}-\vec{k}^{\prime}\right), \\
\left\{b(\vec{k}, r), d\left(\vec{k}^{\prime}, r^{\prime}\right)\right\} & =\left\{b(\vec{k}, r), d\left(\vec{k}^{\prime}, r^{\prime}\right)^{\dagger}\right\}=0 .
\end{aligned}
$$

\section{Charged pion field}

The charged pion fields can be written as

$$
\begin{aligned}
\phi_{\pi^{\sigma}}^{s}(x) & =\phi_{\pi^{-\sigma}}^{s}(x)^{\dagger} \\
& =\sum_{\ell=0}^{\infty} \int \frac{d p^{2} d p^{3}}{(2 \pi)^{3} 2 E_{\pi^{\sigma}}}\left[a^{\sigma}(\breve{p}) \mathbb{F}_{\bar{p}}^{s}(x)+a^{-\sigma}(\breve{p})^{\dagger} \mathbb{F}_{\bar{p}}^{-s}(x)^{*}\right],
\end{aligned}
$$

where $Q_{\pi^{\sigma}}=\sigma|e|$ is the pion charge with $\sigma= \pm, s=$ $\operatorname{sign}\left(Q_{\pi^{\sigma}} B\right)$ and $B_{e}=\left|Q_{\pi^{\sigma}} B\right|$. Note that if $B>0$ then $s=\sigma$. As defined in the main text, $\bar{p}=\left(p^{0}, \breve{p}\right)$, where $\breve{p}=\left(\ell, p^{2}, p^{3}\right)$ and the pion energy is given by $p^{0}=$ $E_{\pi^{\sigma}}=\sqrt{m_{\pi^{\sigma}}^{2}+(2 \ell+1) B_{e}+\left(p^{3}\right)^{2}}$.

The functions $\mathbb{F}_{\bar{p}}^{S}(x)$ are solutions of the eigenvalue equation

$$
\mathcal{D}_{\mu} \mathcal{D}^{\mu} \mathbb{F}_{\bar{p}}^{s}(x)=-\left[\left(p^{0}\right)^{2}-(2 \ell+1) B_{e}-\left(p^{3}\right)^{2}\right] \mathbb{F}_{\bar{p}}^{s}(x),
$$


where $\mathcal{D}^{\mu}=\partial^{\mu}+i s B_{e} x^{1} \delta_{\mu 2}$. Their explicit form is given by

$$
\mathbb{F}_{\bar{p}}^{s}(x)=N_{\ell} e^{-i\left(p^{0} x^{0}-p^{2} x^{2}-p^{3} x^{3}\right)} D_{\ell}\left(\rho_{s}\right),
$$

where $N_{\ell}=\left(4 \pi B_{e}\right)^{1 / 4} / \sqrt{\ell !}, \rho_{s}=\sqrt{2 B_{e}} x^{1}-s \sqrt{2 / B_{e}} p^{2}$ and $D_{\ell}(x)$ are cylindrical parabolic functions. The latter are defined as

$$
D_{\ell}(x)=2^{-\ell / 2} e^{-x^{2} / 4} H_{\ell}(x / \sqrt{2}),
$$

$H_{\ell}(x)$ being the Hermite polynomials, with the standard convention $H_{-1}(x)=0$. It can be seen that the functions $\mathbb{F}_{\bar{p}}^{s}(x)$ satisfy the orthogonality relations

$$
\begin{aligned}
& \sum_{\ell=0}^{\infty} \int \frac{d p^{0} d p^{2} d p^{3}}{(2 \pi)^{4}} \mathbb{F}_{\bar{p}}^{s}(x) \mathbb{F}_{\bar{p}}^{s}\left(x^{\prime}\right)^{*}=\delta^{(4)}\left(x-x^{\prime}\right), \\
& \int d^{4} x \mathbb{F}_{\bar{p}^{\prime}}^{s}(x)^{*} \mathbb{F}_{\bar{p}}^{s}(x)=(2 \pi)^{4} \delta_{\ell \ell^{\prime}} \delta\left(p^{0}-p^{\prime 0}\right) \\
& \times \delta\left(p^{2}-p^{\prime 2}\right) \delta\left(p^{3}-p^{\prime 3}\right) .
\end{aligned}
$$

In addition, the creation and annihilation operators in Eq. (A10) satisfy the commutation relations

$$
\begin{aligned}
{\left[a^{\sigma}(\breve{p}), a^{\sigma}\left(\breve{p}^{\prime}\right)^{\dagger}\right] } & =\left[a^{-\sigma}(\breve{p}), a^{-\sigma}\left(\breve{p}^{\prime}\right)^{\dagger}\right] \\
& =2 E_{\pi^{\sigma}}(2 \pi)^{3} \delta_{\ell \ell^{\prime}} \delta\left(p^{2}-p^{\prime 2}\right) \delta\left(p^{3}-p^{\prime 3}\right) .
\end{aligned}
$$

Note that with these conventions the operators $a^{\sigma}(\breve{p})$ and $a^{-\sigma}(\breve{p})$ turn out to have different dimensions from the creation and annihilation operators that are usually defined in the absence of the external magnetic field [and also from those corresponding to the $\pi^{0}$ field; see Eq. (A2)].

It is also useful to calculate the particle number associated with the state $\left|\pi^{\sigma}(\breve{p})\right\rangle=a^{\dagger}(\breve{p})|0\rangle$ in a volume $V$. Given our choice of the Landau gauge, in this case it is convenient to consider an infinite cylinder of section $S$ lying along the $x^{1}$ axis. We obtain

$$
n_{\pi^{\sigma}}=\int_{-\infty}^{\infty} d x^{1} \int_{S} d x^{2} d x^{3}\left\langle\pi^{\sigma}(\breve{p})\left|j_{\pi^{\sigma}}^{0}(x)\right| \pi^{\sigma}(\breve{p})\right\rangle=2 E_{\pi^{\sigma}} 2 \pi S,
$$

where the current is defined in a similar way to that corresponding to the neutral pion; see Eq. (A4). Note that we are normalizing to $4 \pi E$ particles per unit surface, which differs from the usual normalization $\rho=n / V=2 E$.

\section{Charged lepton field}

Assuming the same conventions for the magnetic field and considering the Landau gauge, for the charged lepton fields we have

$$
\begin{aligned}
\psi_{l}^{s}(x)= & \sum_{r=1,2} \sum_{n=0}^{\infty} \int \frac{d q^{2} d q^{3}}{(2 \pi)^{3} 2 E_{l}}\left[b(\breve{q}, r) U_{l}^{s}(x, \breve{q}, r)\right. \\
& \left.+d(\breve{q}, r)^{\dagger} V_{l}^{-s}(x, \breve{q}, r)\right] .
\end{aligned}
$$

Here, $s=\operatorname{sign}\left(Q_{l} B\right)$ with $Q_{l}=-|e|$. As indicated in the main text, $\breve{q}=\left(n, q^{2}, q^{3}\right)$ and $E_{l}=\sqrt{m_{l}^{2}+2 n B_{e}+\left(q^{3}\right)^{2}}$, with $B_{e}=\left|Q_{l} B\right|$. The creation and annihilation operators satisfy

$$
\begin{aligned}
\left\{b(\breve{q}, r), b\left(\breve{q}^{\prime}, r^{\prime}\right)^{\dagger}\right\}= & \left\{d(\breve{q}, r), d\left(\breve{q}^{\prime}, r^{\prime}\right)^{\dagger}\right\}=2 E_{l}(2 \pi)^{3} \\
& \times \delta_{r r^{\prime}} \delta_{n n^{\prime}} \delta\left(q^{2}-q^{\prime 2}\right) \delta\left(q^{3}-q^{\prime 3}\right), \\
\left\{b(\breve{q}, r), d\left(\breve{q}^{\prime}, r^{\prime}\right)^{\dagger}\right\}= & \left\{d(\breve{q}, r)^{\dagger}, b\left(\breve{q}^{\prime}, r^{\prime}\right)^{\dagger}\right\}=0 .
\end{aligned}
$$

In Eq. (A18) we have also used the definitions

$$
\begin{aligned}
U_{l}^{s}(x, \breve{q}, r) & =\mathbb{E}_{\bar{q}}^{s}(x) u_{l}^{s}(\breve{q}, r), \\
V_{l}^{-s}(x, \breve{q}, r) & =\tilde{\mathbb{E}}_{\bar{q}}^{-s}(x) v_{l}^{-s}(\breve{q}, r),
\end{aligned}
$$

where $\bar{q}=\left(q^{0}, \breve{q}\right)$, with $q^{0}=E_{l}$. The spinors $u_{l}^{s}$ and $v_{l}^{-s}$ are given in the Weyl basis by

$$
\begin{aligned}
u_{l}^{s}(\breve{q}, r)= & \frac{1}{\sqrt{2\left(E_{l}+m_{l}\right)}} \\
& \times\left(\begin{array}{l}
\left(E_{l}+m_{l}+s \sqrt{2 n B_{e}} \tau_{2}-q^{3} \tau_{3}\right) \phi^{(r)} \\
\left(E_{l}+m_{l}-s \sqrt{2 n B_{e}} \tau_{2}+q^{3} \tau_{3}\right) \phi^{(r)}
\end{array}\right),
\end{aligned}
$$

$$
\begin{aligned}
v_{l}^{-s}(\breve{q}, r)= & \frac{1}{\sqrt{2\left(E_{l}+m_{l}\right)}} \\
& \times\left(\begin{array}{c}
\left(E_{l}+m_{l}-s \sqrt{2 n B_{e}} \tau_{2}-q^{3} \tau_{3}\right) \tilde{\phi}^{(r)} \\
-\left(E_{l}+m_{l}+s \sqrt{2 n B_{e}} \tau_{2}+q^{3} \tau_{3}\right) \tilde{\phi}^{(r)}
\end{array}\right),
\end{aligned}
$$

where $\phi^{(1) \dagger}=-\tilde{\phi}^{(2) \dagger}=(1,0)$ and $\phi^{(2) \dagger}=\tilde{\phi}^{(1) \dagger}=(0,1)$. They satisfy the relations

$$
\begin{aligned}
\sum_{r=1,2} u_{l}^{s}(\breve{q}, r) \bar{u}_{l}^{s}(\breve{q}, r) & =\hat{q}_{s}+m_{l}, \\
\sum_{r=1,2} v_{l}^{-s}(\breve{q}, r) \bar{v}_{l}^{-s}(\breve{q}, r) & =\hat{q}_{-s}-m_{l},
\end{aligned}
$$

where $\hat{q}_{s}^{\mu}=\left(E_{l}, 0,-s \sqrt{2 n B_{e}}, q^{3}\right)$. In Eq. (A20), $\mathbb{E}_{\bar{q}}^{s}(x)$ and $\tilde{\mathbb{E}}_{\bar{q}}^{-s}(x)$ are Ritus functions that satisfy the eigenvalue equation

$$
D^{2} \mathbb{E}_{\bar{q}}^{s}(x)=-\left[\left(q^{0}\right)^{2}-2 n B_{e}-\left(q^{3}\right)^{2}\right] \mathbb{E}_{\bar{q}}^{s}(x),
$$

where $D=\not \partial-i s B_{e} x^{1} \gamma^{2}$. They can be written as 
$\mathbb{E}_{\bar{q}}^{s}(x)=\sum_{\lambda= \pm} E_{\bar{q}, \lambda}^{s}(x) \Delta^{\lambda}, \quad \tilde{\mathbb{E}}_{\bar{q}}^{-s}(x)=\sum_{\lambda= \pm} E_{\bar{q},-\lambda}^{-s}(x)^{*} \Delta^{\lambda}$

where $\Delta^{ \pm}=\left(1 \pm i \gamma^{1} \gamma^{2}\right) / 2$, and

$$
E_{\bar{q}, \lambda}^{s}(x)=\mathbb{F}_{\left(q^{0}, n_{s \lambda} \lambda q^{2}, q^{3}\right)}^{s}(x),
$$

$\mathbb{F}_{\bar{q}}^{s}(x)$ being the function defined in Eq. (A12). Here the integer index $n_{s \lambda}$ is related to the quantum number $n$ by

$$
n_{s \pm}=n-\frac{1 \mp s}{2}
$$

It can be seen that the spinors in Eq. (A20) satisfy

$$
\begin{gathered}
\int d^{3} x U^{s \dagger}(x, \breve{q}, r) U^{s}\left(x, \breve{q}^{\prime}, r^{\prime}\right)=\int d^{3} x V^{-s \dagger}(x, \breve{q}, r) V^{-s}\left(x, \breve{q}^{\prime}, r^{\prime}\right)=2 E_{l}(2 \pi)^{3} \delta_{\breve{q}, \breve{q}^{\prime}} \delta_{r r^{\prime}} \\
\int d^{3} x U^{s \dagger}(x, \breve{q}, r) V^{-s}\left(x, \breve{q}^{\prime}, r^{\prime}\right)=\int d^{3} x V^{-s \dagger}(x, \breve{q}, r) U^{s}\left(x, \breve{q}^{\prime}, r^{\prime}\right)=0 \\
\int d^{3} x \bar{U}^{s}(x, \breve{q}, r) U^{s}\left(x, \breve{q}^{\prime}, r^{\prime}\right)=-\int d^{3} x \bar{V}^{-s}(x, \breve{q}, r) V^{-s}\left(x, \breve{q}^{\prime}, r^{\prime}\right)=2 m_{l}(2 \pi)^{3} \delta_{\breve{q}, \breve{q}^{\prime}} \delta_{r r^{\prime}} \\
\int d^{3} x \bar{U}^{s}(x, \breve{q}, r) V^{-s}\left(\tilde{x}, \breve{q}^{\prime}, r^{\prime}\right)=\int d^{3} x \bar{V}^{-s}(x, \breve{q}, r) U^{s}\left(\tilde{x}, \breve{q}^{\prime}, r^{\prime}\right)=0,
\end{gathered}
$$

where $\delta_{\breve{q}, \breve{q}^{\prime}}=\delta_{n n^{\prime}} \delta\left(q^{2}-q^{\prime 2}\right) \delta\left(q^{3}-q^{\prime 3}\right)$ and $\tilde{x}=\left(x^{0},-\vec{x}\right)$.

Following the same steps that led to Eq. (A17), it is found that the number of particles in an infinite cylinder of section $S$ lying along the $x^{1}$ axis is given by $n_{l}=2 E_{l} 2 \pi S$.

\section{APPENDIX B: DISCRETE SYMMETRIES}

In the Landau gauge, the electromagnetic interaction term between the light quarks and the external field (chosen to be orientated along the $z$ axis) is given by

$$
\mathcal{L}(x)=-\sum_{f} Q_{f} B x^{1} \bar{\psi}_{f}(x) \gamma_{2} \psi_{f}(x)
$$

where the sum extends over $f=u, d$, and $Q_{f}$ are the corresponding electric charges. It is easy to see that the action is separately invariant under $\mathcal{P}, \mathcal{C} T$ and $\mathcal{P C T}$, where
$\mathcal{P}, \mathcal{C}$ and $\mathcal{T}$ stand for parity, charge conjugation and time reversal transformations acting on the quark fields. Moreover, it can be seen that the Lagrangian density in Eq. (B1) is invariant under the transformation $\mathcal{C R}_{1}$, where $\mathcal{R}_{1}$ is a spatial rotation by angle $\pi$ about the $x$ axis (i.e., a rotation that inverts the orientation of the magnetic field $\vec{B}$ ).

The existence of these symmetries imposes constraints on the form factors in the pion-to-vacuum hadronic matrix elements discussed in our work. As in the case of no external field, parity is responsible for selecting which Lorentz structures in Eq. (8) contribute to the matrix elements of the vector and axial-vector currents, as quoted in Eqs. (9), (10), (19), and (20). Moreover, it is possible to use $\mathcal{C} \mathcal{T}$ and $\mathcal{C} \mathcal{R}_{1}$ symmetries to show that the form factors are real and equal for both charged pions.

We start by using $\mathcal{C} \mathcal{T}$ symmetry to show that the form factor $f_{\pi^{0}}^{(V)}$ in Eq. (11) is real. One has

$$
\begin{aligned}
\left\langle 0\left|\bar{\psi}_{f}(x) \gamma^{\mu} \psi_{f}(x)\right| \pi^{0}(\vec{p})\right\rangle & =\left\langle 0\left|(\mathcal{C} \mathcal{T})^{\dagger} \mathcal{C} \mathcal{T} \bar{\psi}_{f}(x) \gamma^{\mu} \psi_{f}(x)(\mathcal{C} \mathcal{T})^{\dagger} \mathcal{C} \mathcal{T}\right| \pi^{0}(\vec{p})\right\rangle \\
& =\eta_{T}\left\langle 0\left|\mathcal{C}^{\dagger} \mathcal{C} \bar{\psi}_{f}(-\tilde{x}) \gamma_{\mu} \psi_{f}(-\tilde{x}) \mathcal{C}^{\dagger} \mathcal{C}\right| \pi^{0}(-\vec{p})\right\rangle^{*} \\
& =\left\langle 0\left|\bar{\psi}_{f}(-\tilde{x}) \gamma_{\mu} \psi_{f}(-\tilde{x})\right| \pi^{0}(-\vec{p})\right\rangle^{*}
\end{aligned}
$$

where $\tilde{x}^{\mu}=\left(x^{0},-\vec{x}\right)$, and the phase $\eta_{T}$, arising from the action of the time reversal operator on the pion state, has been taken to be equal to -1 due to $\mathcal{P C T}$ invariance. From Eqs. (11) and (B2) it can be easily seen that $f_{\pi^{0}}^{(V)}$ is real. For example, from the definition of $H_{V}^{0, \mu}(x, \vec{p})$, Eq. (B2) implies $H_{V}^{0,0}(x, \vec{p})=H_{V}^{0,0}(-\tilde{x},-\vec{p})^{*}$, which according to the relations in Eqs. (11) leads to

$$
-i f_{\pi^{0}}^{(V)} p^{3} e^{-i p \cdot x}=\left(-i f_{\pi^{0}}^{(V)}\right)^{*}\left(-p^{3}\right) e^{i \tilde{p} \cdot(-\tilde{x})},
$$

i.e., $f_{\pi^{0}}^{(V)}=f_{\pi^{0}}^{(V) *}$. 
In the case of the matrix elements of the axial vector current, a similar analysis leads to $f_{\pi^{0}}^{(A i)}=f_{\pi^{0}}^{(A i) *}$, for $i=1$, 2 and 3. On the other hand, taking into account the invariance of the action under $\mathcal{C} \mathcal{R}_{1}$ one has

$$
\begin{aligned}
H_{\perp, A}^{0, \epsilon}(x, \vec{p})= & \langle 0|\left(\mathcal{C} \mathcal{R}_{1}\right)^{\dagger} \mathcal{C} \mathcal{R}_{1} \bar{\psi}_{f}(x)\left(\gamma^{1}+i \epsilon \gamma^{2}\right) \\
& \times \gamma_{5} \psi_{f}(x)\left(\mathcal{C} \mathcal{R}_{1}\right)^{\dagger} \mathcal{C} \mathcal{R}_{1}\left|\pi^{0}(\vec{p})\right\rangle \\
= & \left\langle 0\left|\mathcal{C}^{\dagger} \mathcal{C} \bar{\psi}_{f}\left(x^{\prime}\right)\left(\gamma^{1}-i \epsilon \gamma^{2}\right) \gamma_{5} \psi_{f}\left(x^{\prime}\right) \mathcal{C}^{\dagger} \mathcal{C}\right| \pi^{0}\left(\vec{p}^{\prime}\right)\right\rangle \\
= & \left\langle 0\left|\bar{\psi}_{f}\left(x^{\prime}\right)\left(\gamma^{1}-i \epsilon \gamma^{2}\right) \gamma_{5} \psi_{f}\left(x^{\prime}\right)\right| \pi^{0}\left(\vec{p}^{\prime}\right)\right\rangle \\
= & H_{\perp, A}^{0,-\epsilon}\left(x^{\prime}, \vec{p}^{\prime}\right),
\end{aligned}
$$

where $x^{\prime \mu}=\left(x^{0}, x^{1},-x^{2},-x^{3}\right)$ and $\vec{p}^{\prime}=\left(p^{1},-p^{2},-p^{3}\right)$. From Eq. (14), this leads to

$$
\begin{aligned}
& -i\left(f_{\pi^{0}}^{(A 1)}-\epsilon f_{\pi^{0}}^{(A 2)}-f_{\pi^{0}}^{(A 3)}\right)\left(p^{1}+i \epsilon p^{2}\right) e^{-i p \cdot x} \\
& =-i\left(f_{\pi^{0}}^{(A 1)}+\epsilon f_{\pi^{0}}^{(A 2)}-f_{\pi^{0}}^{(A 3)}\right)\left(p^{1}-i \epsilon p^{2}\right) e^{-i p^{\prime} \cdot x^{\prime}},
\end{aligned}
$$

which implies $f_{\pi^{0}}^{(A 2)}=0$.

We consider next the matrix elements with charged pion initial states. Proceeding in a similar way as in the neutral case, from Eq. (19) we get

$$
\begin{aligned}
H_{V}^{\sigma, \mu}(x, \breve{p}) & =\left\langle 0\left|(\mathcal{C} \mathcal{T})^{\dagger} \mathcal{C} \mathcal{T} \bar{\psi}(x) \gamma^{\mu} \tau^{-\sigma} \psi(x)(\mathcal{C} \mathcal{T})^{\dagger} \mathcal{C} \mathcal{T}\right| \pi^{\sigma}(\breve{p})\right\rangle \\
& =-\left\langle 0\left|\mathcal{C}^{\dagger} \mathcal{C} \bar{\psi}(-\tilde{x}) \gamma_{\mu} \tau^{-\sigma} \psi(-\tilde{x}) \mathcal{C}^{\dagger} \mathcal{C}\right| \pi^{\sigma}\left(\breve{p}^{\prime}\right)\right\rangle^{*} \\
& =\left\langle 0\left|\bar{\psi}(-\tilde{x}) \gamma_{\mu} \tau^{\sigma} \psi(-\tilde{x})\right| \pi^{-\sigma}\left(\breve{p}^{\prime}\right)\right\rangle^{*} \\
& =g_{\mu \nu} H_{V}^{-\sigma, \nu}\left(-\tilde{x}, \breve{p}^{\prime}\right)^{*}
\end{aligned}
$$

where we have used $\mathcal{C}\left|\pi^{ \pm}(\bar{p})\right\rangle=\left|\pi^{\mp}(\bar{p})\right\rangle$ and defined $\breve{p}^{\prime}=\left(\ell,-p^{2},-p^{3}\right)$. Since $\mathbb{F}_{\bar{p}}^{\sigma}(x)=\mathbb{F}_{\bar{p}^{\prime}}^{\sigma}(-\tilde{x})^{*}$, taking $\mu=0$ and $\sigma=-$ one obtains [see Eq. (27)]

$$
-i f_{\pi^{-}}^{(V)} p^{3} \mathbb{F}_{\bar{p}}^{-}(x)=\left(-i f_{\pi^{+}}^{(V)} p^{\prime 3} \mathbb{F}_{\bar{p}^{\prime}}^{+}(-\tilde{x})\right)^{*}=-i f_{\pi^{+}}^{(V) *} p^{3} \mathbb{F}_{\bar{p}}^{-}(x),
$$

which leads to the relation $f_{\pi^{+}}^{(V) *}=f_{\pi^{-}}^{(V)}$. Here, $\bar{p}^{\prime}=$ $\left(E_{\pi^{-}}, \breve{p}^{\prime}\right)$. Now, from the invariance of the action under $\mathcal{C R}$ one has

$$
\begin{aligned}
H_{V}^{\sigma, 0}(x, \breve{p})= & \langle 0|\left(\mathcal{C} \mathcal{R}_{1}\right)^{\dagger} \mathcal{C} \mathcal{R}_{1} \bar{\psi}(x) \gamma^{0} \tau^{-\sigma} \psi(x)\left(\mathcal{C} \mathcal{R}_{1}\right)^{\dagger} \\
& \times \mathcal{C} \mathcal{R}_{1}\left|\pi^{\sigma}(\breve{p})\right\rangle \\
= & \left\langle 0\left|\mathcal{C}^{\dagger} \mathcal{C} \bar{\psi}\left(x^{\prime}\right) \gamma^{0} \tau^{-\sigma} \psi\left(x^{\prime}\right) \mathcal{C}^{\dagger} \mathcal{C}\right| \pi^{\sigma}\left(\breve{p}^{\prime}\right)\right\rangle \\
= & -\left\langle 0\left|\bar{\psi}\left(x^{\prime}\right) \gamma^{0} \tau^{\sigma} \psi\left(x^{\prime}\right)\right| \pi^{-\sigma}\left(\breve{p}^{\prime}\right)\right\rangle \\
= & -H_{V}^{-\sigma, 0}\left(x^{\prime}, \breve{p}^{\prime}\right) .
\end{aligned}
$$

Since $\mathbb{F}_{\bar{p}}^{+}(x)=\mathbb{F}_{\bar{p}^{\prime}}^{-}\left(x^{\prime}\right)$, taking $\mu=0$ and $\sigma=-$ one obtains $f_{\pi^{-}}^{(V)}=f_{\pi^{+}}^{(V)}$, and then $\operatorname{Im}\left(f_{\pi^{\sigma}}^{(V)}\right)=0$.

For the matrix elements of the axial vector current, the analysis of the zeroth and third components of the pion-tovacuum amplitude leads to $f_{\pi^{-}}^{(A 1)}=f_{\pi^{+}}^{(A 1)}$ and $\operatorname{Im}\left(f_{\pi^{\sigma}}^{(A 1)}\right)=0$, while to constrain the form factors $f_{\pi^{\sigma}}^{(A 2)}$ and $f_{\pi^{\sigma}}^{(A 3)}$ one needs to study the first and second components. Taking into account the invariance under $\mathcal{C} \mathcal{T}$ one has $(\epsilon= \pm)$

$$
\begin{aligned}
H_{\perp, A}^{\sigma, \epsilon}(x, \breve{p}) & =\left\langle 0\left|(\mathcal{C} \mathcal{T})^{\dagger} \mathcal{C} \mathcal{T} \bar{\psi}(x)\left(\gamma^{1}+i \epsilon \gamma^{2}\right) \gamma_{5} \tau^{-\sigma} \psi(x)(\mathcal{C} \mathcal{T})^{\dagger} \mathcal{C} \mathcal{T}\right| \pi^{\sigma}(\breve{p})\right\rangle \\
& =-\left\langle 0\left|\mathcal{C}^{\dagger} \mathcal{C} \bar{\psi}(-\tilde{x})\left(\gamma_{1}-i \epsilon \gamma_{2}\right) \gamma_{5} \tau^{-\sigma} \psi(-\tilde{x}) \mathcal{C}^{\dagger} \mathcal{C}\right| \pi^{\sigma}\left(\breve{p}^{\prime}\right)\right\rangle^{*} \\
& =+\left\langle 0\left|\bar{\psi}(-\tilde{x})\left(\gamma^{1}-i \epsilon \gamma^{2}\right) \gamma_{5} \tau^{\sigma} \psi(-\tilde{x})\right| \pi^{-\sigma}\left(\breve{p}^{\prime}\right)\right\rangle^{*} \\
& =\left(H_{A}^{-\sigma, 1}\left(-\tilde{x}, \bar{p}^{\prime}\right)-i \epsilon H_{A}^{-\sigma, 2}\left(-\tilde{x}, \breve{p}^{\prime}\right)\right)^{*}=\left(H_{\perp, A}^{-\sigma,-\epsilon}\left(-\tilde{x}, \breve{p}^{\prime}\right)\right)^{*}
\end{aligned}
$$

In this way, taking $\sigma=-$ in Eqs. (30) and (B9) one obtains

$$
\left(f_{\pi^{-}}^{(A 1)}+\epsilon f_{\pi^{-}}^{(A 2)}-f_{\pi^{-}}^{(A 3)}\right) \mathbb{F}_{\bar{p}+\epsilon}^{-}(x)=\left(f_{\pi^{+}}^{(A 1)}+\epsilon f_{\pi^{+}}^{(A 2)}-f_{\pi^{+}}^{(A 3)}\right)^{*} \mathbb{F}_{\bar{p}^{\prime}+\epsilon}^{+}(-\tilde{x})^{*},
$$

which implies $f_{\pi^{-}}^{(A 2)}=f_{\pi^{+}}^{(A 2) *}$ and $f_{\pi^{-}}^{(A 3)}=f_{\pi^{+}}^{(A 3) *}$ (we have used the fact that $f_{\pi^{-}}^{(A 1)}=f_{\pi^{+}}^{(A 1) *}$ ). Finally, considering $\mathcal{C} \mathcal{R}_{1}$ transformations, one has

$$
\begin{aligned}
H_{\perp, A}^{\sigma, \epsilon}(x, \breve{p}) & =\left\langle 0\left|\left(\mathcal{C} \mathcal{R}_{1}\right)^{\dagger} \mathcal{C} \mathcal{R}_{1} \bar{\psi}(x)\left(\gamma^{1}+i \epsilon \gamma^{2}\right) \gamma_{5} \tau^{-\sigma} \psi(x)\left(\mathcal{C} \mathcal{R}_{1}\right)^{\dagger} \mathcal{C} \mathcal{R}_{1}\right| \pi^{\sigma}(\breve{p})\right\rangle \\
& =\left\langle 0\left|\mathcal{C}^{\dagger} \mathcal{C} \bar{\psi}\left(x^{\prime}\right)\left(\gamma^{1}-i \epsilon \gamma^{2}\right) \gamma_{5} \tau^{-\sigma} \psi\left(x^{\prime}\right) \mathcal{C}^{\dagger} \mathcal{C}\right| \pi^{\sigma}\left(\breve{p}^{\prime}\right)\right\rangle \\
& =\left\langle 0\left|\bar{\psi}\left(x^{\prime}\right)\left(\gamma^{1}-i \epsilon \gamma^{2}\right) \gamma_{5} \tau^{\sigma} \psi\left(x^{\prime}\right)\right| \pi^{-\sigma}\left(\breve{p}^{\prime}\right)\right\rangle \\
& =H_{A}^{-\sigma, 1}\left(x^{\prime}, \breve{p}^{\prime}\right)-i \epsilon H_{A}^{-\sigma, 2}\left(x^{\prime}, \breve{p}^{\prime}\right)=H_{\perp, A}^{-\sigma, \epsilon}\left(x^{\prime}, \breve{p}^{\prime}\right),
\end{aligned}
$$

which leads to $f_{\pi^{-}}^{(A 2)}=f_{\pi^{+}}^{(A 2)}$ and $f_{\pi^{-}}^{(A 3)}=f_{\pi^{+}}^{(A 3)}$, together with $\operatorname{Im}\left(f_{\pi^{\sigma}}^{(A 2)}\right)=\operatorname{Im}\left(f_{\pi^{\sigma}}^{(A 3)}\right)=0$. 


\section{APPENDIX C: SPATIAL INTEGRAL}

We are interested in the calculation of the integral

$$
I_{\breve{p}, \breve{q}, \vec{k}}^{s, \lambda}=\int d^{4} x \mathbb{F}_{\bar{p}}^{s}(x) E_{\bar{q}, \lambda}^{s}(x)^{*} e^{i k \cdot x},
$$

where $s, \lambda= \pm$. Here, $\mathbb{F}_{\bar{p}}^{s}(x)$ comes from the pion, $e^{i k \cdot x}$ from the neutrino and $E_{\bar{q}, \lambda}^{s}(x)$ from the lepton. We use the notation already defined in Appendix A, namely $\bar{q}=\left(E_{l}, \breve{q}\right)$ and $\bar{p}=\left(E_{\pi^{-}}, \breve{p}\right)$, with $\breve{q}=\left(n, q^{2}, q^{3}\right)$ and $\breve{p}=\left(\ell, p^{2}, p^{3}\right)$, respectively. Since $\left|Q_{\pi^{ \pm}} B\right|=\left|Q_{l} B\right|=|e B|=B_{e}$ and we are interested in the situation where $\operatorname{sign}\left(Q_{\pi^{ \pm}} B\right)=$ $\operatorname{sign}\left(Q_{l} B\right)=s$, after integration over $x^{0}, x^{2}$ and $x^{3}$ we obtain

$$
I_{\breve{p}, \breve{q}, \vec{k}}^{s, \lambda}=(2 \pi)^{3} \delta\left(E_{\pi^{-}}-E_{\nu_{l}}-E_{l}\right) \delta\left(p^{2}-k^{2}-q^{2}\right) \delta\left(p^{3}-k^{3}-q^{3}\right) \mathcal{I}_{\ell, n}^{s, \lambda}\left(k_{\perp}, p^{2}, q^{2}\right),
$$

with

$$
\mathcal{I}_{\ell, n}^{s, \lambda}\left(k_{\perp}, p^{2}, q^{2}\right)=N_{\ell} N_{n_{s \lambda}} \int_{-\infty}^{\infty} d x^{1} e^{-i k^{1} x^{1}} D_{\ell}\left(\sqrt{2 B_{e}} x^{1}-s \sqrt{\frac{2}{B_{e}}} p^{2}\right) D_{n_{s \lambda}}\left(\sqrt{2 B_{e}} x^{1}-s \sqrt{\frac{2}{B_{e}}} q^{2}\right),
$$

where, as also defined in Appendix A, $n_{s \lambda}=n+(s \lambda-1) / 2$. Changing variables according to

$$
\left\{\begin{array}{l}
\psi=\sqrt{2 B_{e}} x^{1}-\frac{s}{\sqrt{2 B_{e}}}\left(p^{2}+q^{2}\right) \\
\eta=\frac{s}{\sqrt{2 B_{e}}}\left(p^{2}-q^{2}\right)
\end{array}, \quad\left\{\begin{array}{l}
\sqrt{2 B_{e}} x^{1}-s \sqrt{\frac{2}{B_{e}}} p^{2}=\psi-\eta \\
\sqrt{2 B_{e}} x^{1}-s \sqrt{\frac{2}{B_{e}}} q^{2}=\psi+\eta
\end{array}\right.\right.
$$

and using $D_{\ell}(\psi-\eta)=(-1)^{\ell} D_{\ell}(\eta-\psi)$, we find

$$
\mathcal{I}_{\ell, n}^{s, \lambda}\left(k_{\perp}, p^{2}, q^{2}\right)=N_{\ell} N_{n_{s \lambda}} \frac{(-1)^{\ell}}{\sqrt{2 B_{e}}} e^{-i \frac{s k^{1}\left(p^{2}+q^{2}\right)}{2 B_{e}}} \int_{-\infty}^{\infty} d \psi e^{-i \frac{k^{1}}{\sqrt{2 B_{e}}} \psi} D_{\ell}(\eta-\psi) D_{n_{s \lambda}}(\eta+\psi) .
$$

Next, we make use of the following property:

$$
\int d \psi e^{i \gamma \psi} D_{\ell}(\eta-\psi) D_{n}(\eta+\psi)= \begin{cases}(-1)^{\ell} \sqrt{2 \pi} \ell ! e^{-\frac{\gamma^{2}+\eta^{2}}{2}}(i \gamma+\eta)^{n-\ell} L_{\ell}^{n-\ell}\left(\eta^{2}+\gamma^{2}\right) & \text { if } n \geq \ell \\ (-1)^{n} \sqrt{2 \pi} n ! e^{-\frac{\gamma^{2}+\eta^{2}}{2}}(-i \gamma+\eta)^{\ell-n} L_{n}^{\ell-n}\left(\eta^{2}+\gamma^{2}\right) & \text { if } \ell \geq n .\end{cases}
$$

In our case, $\gamma=-k^{1} / \sqrt{2 B_{e}}$. Owing to one of the delta functions in Eq. (C2), one has $\eta=s k^{2} / \sqrt{2 B_{e}}$ and therefore $\eta^{2}+\gamma^{2}=k_{\perp}^{2} / 2 B_{e}$. Then,

$$
\mathcal{I}_{\ell, n}^{s, \lambda}\left(k_{\perp}, p^{2}, q^{2}\right)=N_{\ell} N_{n_{s \lambda}} \sqrt{\frac{\pi}{B_{e}}} e^{-i s k^{1}\left(p^{2}+q^{2}\right) /\left(2 B_{e}\right)} e^{-k_{\perp}^{2} /\left(4 B_{e}\right)} \begin{cases}\ell !\left(\frac{-i k^{1}+s k^{2}}{\sqrt{2 B_{e}}}\right)^{n_{s \lambda}-\ell} L_{\ell}^{n_{s \lambda}-\ell}\left(\frac{k_{\perp}^{2}}{2 B_{e}}\right) & \text { if } n_{s \lambda} \geq \ell \\ n_{s \lambda} !\left(\frac{i k^{1}+s k^{2}}{\sqrt{2 B_{e}}}\right)^{\ell-n_{s \lambda}} L_{n_{s \lambda}}^{\ell-n_{s \lambda}}\left(\frac{k_{\perp}^{2}}{2 B_{e}}\right) & \text { if } \ell \geq n_{s \lambda} .\end{cases}
$$

Writing the explicit expression of $N_{\ell}, N_{n_{s \lambda}}$ and canceling some terms, we finally arrive at

$$
\mathcal{I}_{\ell, n}^{s, \lambda}\left(k_{\perp}, p^{2}, q^{2}\right)=e^{-i s k^{1}\left(p^{2}+q^{2}\right) /\left(2 B_{e}\right)} \mathcal{G}_{\ell, n}^{s, \lambda}\left(k_{\perp}\right),
$$

where

$$
\mathcal{G}_{\ell, n}^{s, \lambda}\left(k_{\perp}\right)=2 \pi e^{-\frac{k_{\perp}^{2}}{4 B_{e}}} \begin{cases}\sqrt{\frac{\ell !}{n_{s \lambda} !}}\left(\frac{-i k^{1}+s k^{2}}{\sqrt{2 B_{e}}}\right)^{n_{s \lambda}-\ell} L_{\ell}^{n_{s \lambda}-\ell}\left(\frac{k_{\perp}^{2}}{2 B_{e}}\right) & \text { if } n_{s \lambda} \geq \ell \\ \sqrt{\frac{n_{s \lambda} !}{\ell !}}\left(\frac{-i k^{1}-s k^{2}}{\sqrt{2 B_{e}}}\right)^{\ell-n_{s \lambda}} L_{n_{s \lambda}}^{\ell-n_{s \lambda}}\left(\frac{k_{\perp}^{2}}{2 B_{e}}\right) & \text { if } \ell \geq n_{s \lambda} .\end{cases}
$$




\section{APPENDIX D: $B \rightarrow 0$ LIMIT}

We provide here the explicit calculation of the integrals $\mathcal{I}_{0, n}^{-, \lambda}$ appearing in Eq. (73). Let us consider the integral in Eq. (C3) extended to a finite length, in the limit of a weak external magnetic field. We find it convenient to define

$$
\begin{aligned}
\mathcal{I}_{\ell, n}^{-, \lambda}= & N_{\ell} N_{n_{-\lambda}} \int_{-L}^{L} d x^{1} e^{i k^{1} x^{1}} D_{\ell}\left(\sqrt{2 B_{e}} x^{1}\right) \\
& \times D_{n_{-\lambda}}\left(\sqrt{2 B_{e}} x^{1}+\sqrt{\frac{2}{B_{e}}} q^{2}\right)
\end{aligned}
$$

where we have taken $p^{2}=0$, and $L$ is assumed to satisfy the conditions in Eqs. (67) and (68). It is worth noticing that $N_{\ell} D_{\ell}\left(\sqrt{2 B_{e}} x^{1}\right)$ comes form the pion field, $N_{n_{-\lambda}} D_{n_{-\lambda}}\left(\sqrt{2 B_{e}} x^{1}+\sqrt{\left(2 / B_{e}\right)} q^{2}\right)$ comes from the lepton field, and the exponential comes from the antineutrino field.

As discussed in Sec. III D, in the $B \rightarrow 0$ limit one has $\sqrt{2 B_{e}} x^{1} \rightarrow 0$; therefore the $\ell=0$ and $\ell=1$ pion wave functions satisfy

$$
\begin{aligned}
& N_{0} D_{0}\left(\sqrt{2 B_{e}} x^{1}\right) \underset{\sqrt{2 B_{e}} x^{1} \rightarrow 0}{\longrightarrow}\left(4 \pi B_{e}\right)^{1 / 4}, \\
& N_{1} D_{1}\left(\sqrt{2 B_{e}} x^{1}\right) \underset{\sqrt{2 B_{e}} x^{1} \rightarrow 0}{\longrightarrow}\left(4 \pi B_{e}\right)^{1 / 4} \frac{1}{2} \sqrt{2 B_{e}} x^{1} \sim 0 .
\end{aligned}
$$

For the lepton contribution, we have to analyze the behavior of the product $N_{n_{-\lambda}} D_{n_{-\lambda}}\left(\sqrt{2 B_{e}} x^{1}+\sqrt{\left(2 / B_{e}\right)} q^{2}\right)$ in the limit of small $B$ and large $n$, keeping $n B$ finite. Using Eqs. (12.7.2), (12.10.35), (9.7.5) and (9.7.9) of Ref. [37] we obtain

$$
\begin{aligned}
& \frac{\left(4 \pi B_{e}\right)^{1 / 4}}{\sqrt{n_{-\lambda} !}} D_{n_{-\lambda}}\left(\sqrt{2 B_{e}} x^{1}+\sqrt{\frac{2}{B_{e}}} q^{2}\right) \\
& \simeq \theta\left(1-r_{n_{-\lambda}}\right)\left[(-1)^{n_{-\lambda}} \theta\left(-q^{2}\right)+\theta\left(q^{2}\right)\right] \\
& \quad \times \frac{\sqrt{B_{e}}}{\left|\left(q^{2}\right)^{2}-B_{e}\left(2 n_{-\lambda}+1\right)\right|^{1 / 4}} \\
& \quad \times\left[e^{i\left(\phi_{n_{-\lambda}}-q_{n_{-\lambda}} x^{1}\right)}+e^{-i\left(\phi_{n_{-\lambda}}-q_{n_{-\lambda}} x^{1}\right)}\right],
\end{aligned}
$$

where

$$
\begin{aligned}
\phi_{n_{-\lambda}} & =\left[\frac{\left(2 n_{-\lambda}+1\right)}{2}\left(\arccos r_{n_{-\lambda}}-r_{n_{-\lambda}} \sqrt{1-r_{n_{-\lambda}}^{2}}\right)-\frac{\pi}{4}\right] \\
q_{n_{-\lambda}} & =\frac{\left|q^{2}\right|}{q^{2}} \sqrt{B_{e}\left(2 n_{-\lambda}+1\right)-\left(q^{2}\right)^{2}} \\
r_{n_{-\lambda}} & =\frac{\left|q^{2}\right|}{\sqrt{B_{e}\left(2 n_{-\lambda}+1\right)}}
\end{aligned}
$$

Here, all quantities have a smooth behavior in the limit of small $B$, large $n$ and finite $n B$, except $\phi_{n_{-\lambda}}$.

The above equations lead to

$$
\begin{aligned}
\mathcal{I}_{0, n}^{-, \lambda}= & 2 \pi\left(4 \pi B_{q}\right)^{1 / 4} \theta\left(1-\frac{\left|q^{2}\right|}{\sqrt{B_{e}\left(2 n_{-\lambda}+1\right)}}\right) \\
& \times\left[(-1)^{n_{-\lambda}} \theta\left(-q^{2}\right)+\theta\left(q^{2}\right)\right] \\
& \times \frac{\sqrt{B_{e}}}{\left|\left(q^{2}\right)^{2}-B_{e}\left(2 n_{-\lambda}+1\right)\right|^{1 / 4}} \\
& \times\left[e^{i \phi_{n_{-\lambda}}} \delta\left(k^{1}-q_{n_{-\lambda}}\right)+e^{-i \phi_{n_{-\lambda}}} \delta\left(k^{1}+q_{n_{-\lambda}}\right)\right],
\end{aligned}
$$

from which we can easily calculate the quantities $\left|\mathcal{I}_{0, n}^{-, \lambda}\right|^{2}$ appearing in Eq. (73). We are also interested in the product $\mathcal{I}_{0, n}^{-,+}\left(\mathcal{I}_{0, n}^{-,-}\right)^{*}$, which involves the divergent phases $\phi_{n_{-}}$and $\phi_{n_{+}}$. These appear through the finite difference

$$
\phi_{n_{-+}}-\phi_{n_{--}} \simeq-\arccos \frac{\left|q^{2}\right|}{\sqrt{2 n B_{e}}},
$$

which leads to

$$
e^{i\left(\phi_{n_{-+}}-\phi_{n_{--}}\right)}=\frac{\left|q^{2}\right|+i \bar{q}_{n}}{\sqrt{2 n B_{e}}}
$$

with $\bar{q}_{n}=\sqrt{2 n B_{e}-\left(q^{2}\right)^{2}}$. We obtain in this way

$$
\begin{aligned}
& \mathcal{I}_{0, n}^{-,+}\left(\mathcal{I}_{0, n}^{-,-}\right)^{*} \\
& \simeq 4 \pi L\left(4 \pi B_{e}\right)^{1 / 2} \theta\left(1-\frac{\left|q^{2}\right|}{\sqrt{2 n B_{e}}}\right) \frac{B_{e}}{\bar{q}_{n}} \frac{1}{\sqrt{2 n B_{e}}} \\
& \quad \times\left\{\left[-\theta\left(-q^{2}\right)\left(-q^{2}+i \bar{q}_{n}\right)+\theta\left(q^{2}\right)\left(q^{2}-i \bar{q}_{n}\right)\right] \delta\left(k^{1}+\bar{q}_{n}\right)\right. \\
& \left.\quad+\left[-\theta\left(-q^{2}\right)\left(-q^{2}-i \bar{q}_{n}\right)+\theta\left(q^{2}\right)\left(q^{2}+i \bar{q}_{n}\right)\right] \delta\left(k^{1}-\bar{q}_{n}\right)\right\} .
\end{aligned}
$$

[1] D. E. Kharzeev, K. Landsteiner, A. Schmitt, and H. U. Yee, Lect. Notes Phys. 871, 1 (2013).

[2] J. O. Andersen, W. R. Naylor, and A. Tranberg, Rev. Mod. Phys. 88, 025001 (2016).
[3] V. A. Miransky and I. A. Shovkovy, Phys. Rep. 576, 1 (2015).

[4] D. Grasso and H. R. Rubinstein, Phys. Rep. 348, 163 (2001). 
[5] D. E. Kharzeev, L. D. McLerran, and H. J. Warringa, Nucl. Phys. A803, 227 (2008); V. Skokov, A. Y. Illarionov, and V. Toneev, Int. J. Mod. Phys. A 24, 5925 (2009); V. Voronyuk, V. Toneev, W. Cassing, E. Bratkovskaya, V. Konchakovski, and S. Voloshin, Phys. Rev. C 83, 054911 (2011).

[6] R. C. Duncan and C. Thompson, Astrophys. J. 392, L9 (1992); C. Kouveliotou et al., Nature (London) 393, 235 (1998).

[7] V. P. Gusynin, V. A. Miransky, and I. A. Shovkovy, Phys. Rev. Lett. 73, 3499 (1994); 76, 1005(E) (1996).

[8] G. S. Bali, F. Bruckmann, G. Endrődi, Z. Fodor, S. D. Katz, S. Krieg, A. Schafer, and K. K. Szabo, J. High Energy Phys. 02 (2012) 044; G. S. Bali, F. Bruckmann, G. Endrődi, Z. Fodor, S. D. Katz, and A. Schafer, Phys. Rev. D 86, 071502 (2012).

[9] A. I. Nikishov and V. I. Ritus, Zh. Eksp. Teor. Fiz. 46, 776 (1964) [Sov. Phys. JETP 19, 529 (1964)].

[10] A. I. Nikishov and V. I. Ritus, Zh. Eksp. Teor. Fiz. 46, 1768 (1964) [Sov. Phys. JETP 19, 1191 (1964)].

[11] J. J. Matese and R. F. O'Connell, Phys. Rev. 180, 1289 (1969).

[12] L. Fassio-Canuto, Phys. Rev. 187, 2141 (1969).

[13] N. O. Agasian and I. A. Shushpanov, J. High Energy Phys. 10 (2001) 006.

[14] J. O. Andersen, J. High Energy Phys. 10 (2012) 005.

[15] S. Fayazbakhsh, S. Sadeghian, and N. Sadooghi, Phys. Rev. D 86, 085042 (2012).

[16] S. S. Avancini, W. R. Tavares, and M. B. Pinto, Phys. Rev. D 93, 014010 (2016).

[17] R. Zhang, W.j. Fu, and Y.x. Liu, Eur. Phys. J. C 76, 307 (2016).

[18] S. S. Avancini, R. L. S. Farias, M. Benghi Pinto, W. R. Tavares, and V. S. Timóteo, Phys. Lett. B 767, 247 (2017).

[19] S. Mao and Y. Wang, Phys. Rev. D 96, 034004 (2017).

[20] D. Gomez Dumm, M. F. Izzo Villafañe, and N. N. Scoccola, Phys. Rev. D 97, 034025 (2018).
[21] M. A. Andreichikov and Y. A. Simonov, Eur. Phys. J. C 78, 902 (2018).

[22] V. D. Orlovsky and Y. A. Simonov, J. High Energy Phys. 09 (2013) 136.

[23] M. A. Andreichikov, B. O. Kerbikov, E. V. Luschevskaya, Y. A. Simonov, and O. E. Solovjeva, J. High Energy Phys. 05 (2017) 007.

[24] Z. Wang and P. Zhuang, Phys. Rev. D 97, 034026 (2018).

[25] H. Liu, X. Wang, L. Yu, and M. Huang, Phys. Rev. D 97, 076008 (2018).

[26] M. Coppola, D. Gomez Dumm, and N. N. Scoccola, Phys. Lett. B 782, 155 (2018).

[27] E. V. Luschevskaya, O. E. Solovjeva, O. A. Kochetkov, and O. V. Teryaev, Nucl. Phys. B898, 627 (2015).

[28] G. S. Bali, B. B. Brandt, G. Endrődi, and B. Gläßle, Phys. Rev. D 97, 034505 (2018).

[29] S. Fayazbakhsh and N. Sadooghi, Phys. Rev. D 88, 065030 (2013).

[30] Y. A. Simonov, Yad. Fiz. 79, 277 (2016) [Phys. At. Nucl. 79, 455 (2016)].

[31] G. S. Bali, B. B. Brandt, G. Endrődi, and B. Gläßle, Phys. Rev. Lett. 121, 072001 (2018).

[32] M. Tanabashi et al. (Particle Data Group), Phys. Rev. D 98, 030001 (2018).

[33] W. Dittrich and H. Gies, Springer Tracts Mod. Phys. 166, 1 (2000).

[34] M. Wakamatsu, Y. Kitadono, and P.-M. Zhang, Ann. Phys. (Amsterdam) 392, 287 (2018).

[35] A. A. Sokolov, I. M. Ternov, and C. W. Kilmister, Radiation from Relativistic Electrons (AIP, New York, 1986).

[36] M. E. Peskin and D. V. Schroeder, An Introduction to Quantum Field Theory (Westview Press, Reading, 1995).

[37] Digital library of mathematical functions, NIST, https://dlmf .nist.gov. 